\title{
Treatment of an Endo-Perio Lesion with Ozone Gas in a Patient with Aggressive Periodontitis: A Clinical Case Report and Literature Review
}

This article was published in the following Dove Press journal: Clinical, Cosmetic and Investigational Dentistry

\author{
Maria K Makeeva $\mathbb{D}^{1,2}$ \\ Fatima Yu Daurova \\ Svetlana F Byakova ${ }^{2}$ \\ Anna Yu Turkina $\mathbb{D}^{2}$
}

'Conservative Dentistry Department, People's Friendship University of Russia (RUDN University), Moscow, Russia; ${ }^{2}$ Therapeutic Dentistry Department, I.M. Sechenov First Moscow State Medical University (Sechenov University), Moscow, Russia
Correspondence: Maria K Makeeva Conservative Dentistry Department, Peoples Friendship University of Russia (RUDN University), Mikluho-Maklaya Street 10-2, Room 267, Moscow 117198 , Russia

Tel +79057416437

Email abrek93@mail.ru

\begin{abstract}
The pulp and periodontium have obvious relationships that have been described in many studies. Pulp infections may affect periodontal tissues and vice versa. Teeth with endo-perio lesions have a worse prognosis than isolated endodontic or periodontal lesions. Elimination of endodontic and periodontal infections is essential for successful treatment, so co-operation between endodontists and periodontists is necessary. In this clinical case, a 44year-old male presented with primary periodontal disease with secondary endodontic involvement in his lower right canine because of aggressive periodontitis. There was $10 \mathrm{~mm}$ of clinical attachment loss and $8 \mathrm{~mm}$ periodontal pocket mesial from the tooth and bone radiolucency periapical and lateral from the root. Periodontal therapy was followed by endodontic treatment. Periodontal therapy included root scaling and planing, treatment of the periodontal pocket with ozone gas, systemic antibiotics, oral hygiene instructions, and chlorhexidine rinsing. Endodontic therapy included root canal instrumentation with rotary endodontic files, irrigation, root canal treatment with ozone gas, and obturation with lateral compaction. Radiographs at a 6-month follow-up appointment showed complete healing of the periapical lesion and alveolar bone lateral to the root. Using an interdisciplinary approach to treat endo-perio lesions provides favorable clinical outcomes. Ozone therapy is beneficial for the successful treatment of endo-perio lesions with narrow periodontal pockets in patients with aggressive periodontitis and poor prognosis.
\end{abstract}

Keywords: endo-perio lesion, ozone therapy, ozone gas

\section{Introduction}

The pulp and periodontal interrelationships have been widely investigated by many authors. ${ }^{1-3}$ Root canals and periodontal pockets contain physiological communications such as apical foramen, lateral canals foramina, and dentinal tubules. These anatomical structures may become pathways for the migration of periodontal and endodontic pathogens and contribute to the development of endo-perio lesions. ${ }^{4,5}$

Endo-perio lesions are challenging to diagnose and treat, causing variable prognosis. Treating these lesions is a complicated task with unpredictable outcomes. Song et $\mathrm{al}^{6}$ compared the clinical outcomes between isolated endodontic lesions and endodontic-periodontal combined lesions and concluded that the latter had a negative effect on clinical outcomes. The prognosis for such lesions is considered poor and mainly depends on the severity of the periodontal involvement. ${ }^{7}$

Generally, extracting teeth with a poor prognosis and replacing them with implants is reasonable. However, a previous history of periodontal disease should 
be evaluated, and, if present, it can be considered a risk factor for future peri-implant disease. ${ }^{8}$ Periodontal diseases such as aggressive periodontitis negatively affect implant osseointegration. ${ }^{9}$ Thus, clinicians should save such teeth rather than extract them and replace them with implants. ${ }^{10}$ Comparing the survival rate between endodontically treated teeth with poor prognosis and implants placed in previously diseased sites showed that the survival rate of endodontically treated teeth was comparatively higher than implants. ${ }^{11}$

Several classifications have been proposed for endoperio lesions. ${ }^{4,-12-15}$ There is no universal international classification of endo-perio lesions, so evaluating the literature data may be problematic because of the diversity of terminology. Rotstein and Simon's ${ }^{4}$ classification was used for the present case. This classification categorizes primary endodontic diseases, primary periodontal diseases, and combined diseases, including primary endodontic disease with secondary periodontal involvement (PESP), primary periodontal disease with secondary endodontic involvement (PPSE), and true combined diseases. Using this classification provides valuable input for sound clinical decisions. $^{16}$

This case provides an example of an interdisciplinary approach between the endodontist and periodontist for the successful treatment of an endo-perio lesion in a patient with aggressive periodontitis in the lower right canine using ozone therapy for periodontal pocket and root canal treatment.

\section{Case Report}

A 44-year-old male with non-contributory medical, family, and psychological history presented at the Conservative Dentistry Department of Sechenov University and reported bleeding during tooth brushing, frontal lower teeth mobility, and episodic suppuration of the gingiva. The patient also reported with the following history: first signs of gum bleeding started at puberty, at the age of 35 he had already had his frontal teeth mobile, tooth 46 was extracted because of mobility and alveolar bone loss. The patient had no systemic pathology. The patient signed written informed consent for treatment and for publishing of case details and images. Institutional approval First Moscow State University, named after I.M. Sechenov (Sechenov University), for case publication was obtained. Clinical examination revealed pus discharge (Figure $1 \mathrm{~A}$ and $\mathrm{B}$ ) and gingival swelling, $82.2 \%$ of the sites had a probing depth (PD) of $\geq 4 \mathrm{~mm}$, and $87 \%$ of the sites had bleeding

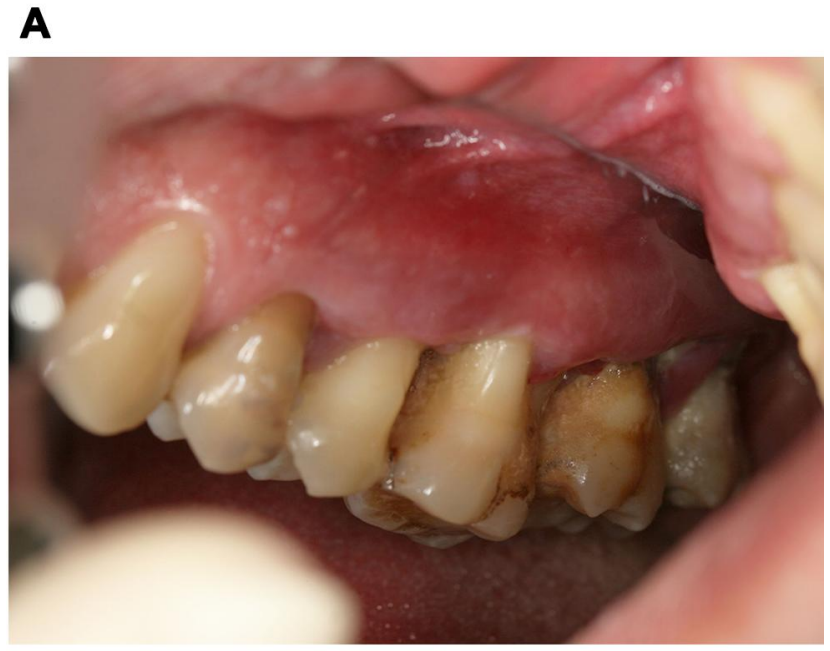

B

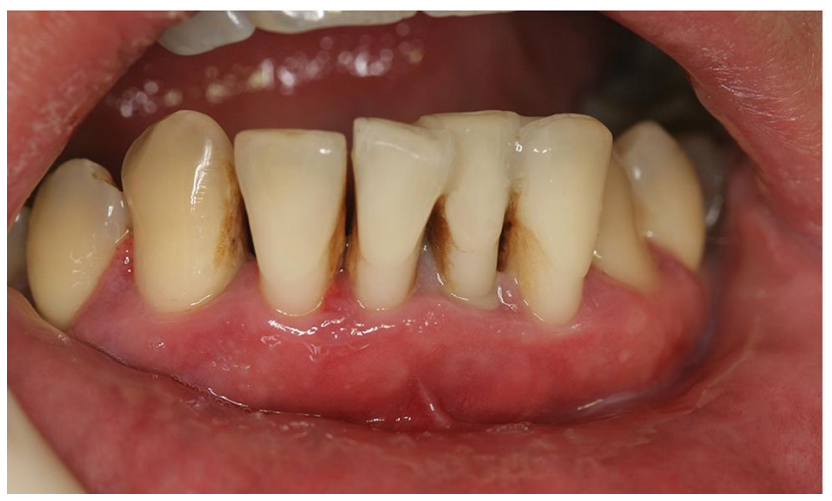

Figure I (A) Intraoral view of the upper right quadrant at baseline; calculus and gum bleeding is visible. (B) Intraoral view of the frontal lower teeth at baseline; small amounts of calculus and pus discharge are visible.

on probing. Oral Hygiene Index-Simplified ${ }^{17}$ had a score of 1.4. The oral hygiene level was defined as moderate and inconsistent with the degree of attachment loss. Panoramic radiographs were obtained and revealed horizontal and vertical alveolar bone resorption (Figure 2).

In the area of teeth \#43 and \#34 (according to the International Dental Federation tooth notation ISO 3950), ${ }^{18}$ alveolar bone resorption reached the apical third of the root. Both teeth were responsive to the cold test. Sensitivity after the test stopped within 10 seconds. The electric pulp test confirmed the vitality of both teeth. Tooth 43 presented II degree of mobility.

The diagnosis was generalized aggressive periodontitis. The patient was prescribed systemic antibiotic ciprofloxacin $500 \mathrm{mg}$ b.i.d. for 7 days. There was no pus discharge after antibiotic therapy (Figure $3 \mathrm{~A}$ and $\mathrm{B}$ ).

The patient underwent thorough scaling and root planing (SRP), splinting of the mobile teeth, occlusal checking and adjustment was done after splinting of frontal teeth to avoid 


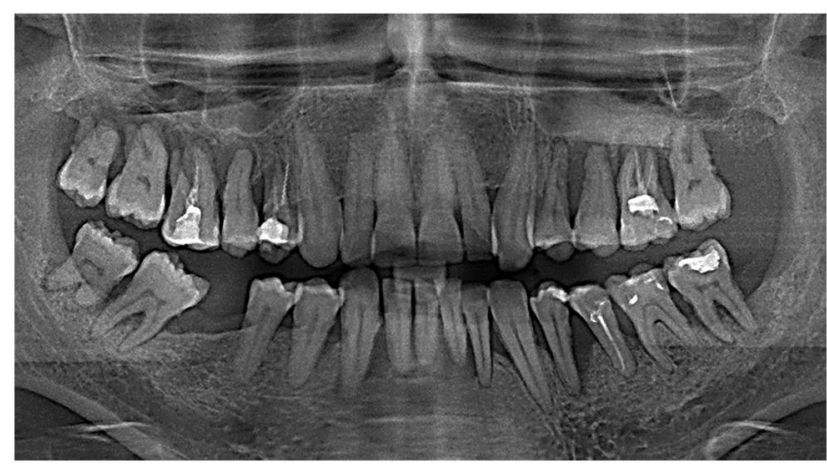

Figure 2 Panoramic radiograph at baseline.

A

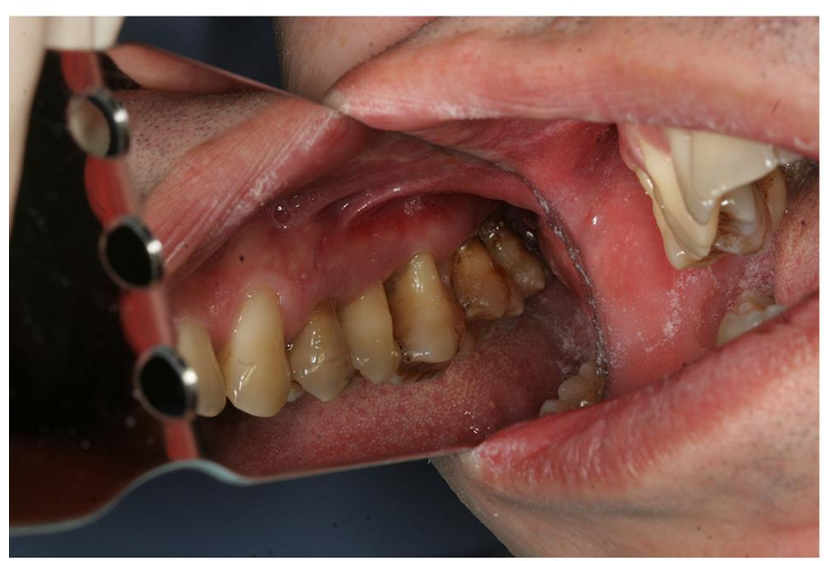

B

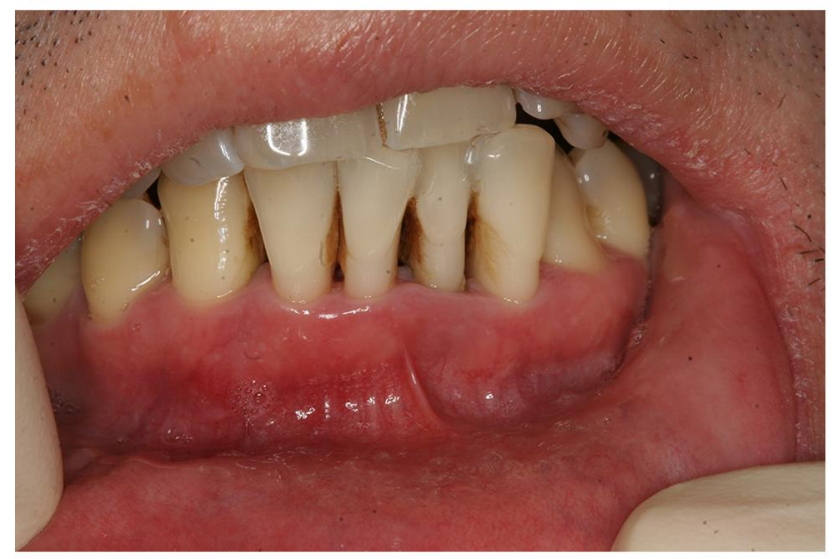

Figure 3 (A) Upper right quadrant after antibiotic therapy. (B) Frontal lower teeth after antibiotic therapy.

trauma from occlusion, and home oral hygiene instructions and training; $0.12 \%$ chlorhexidine rinsing was prescribed twice daily for 14 days. Follow-up 2 weeks after RSP showed no pus or bleeding on probing (Figure 4). The patient adhered to a strict maintenance program with monthly appointments with the same dental hygienist.

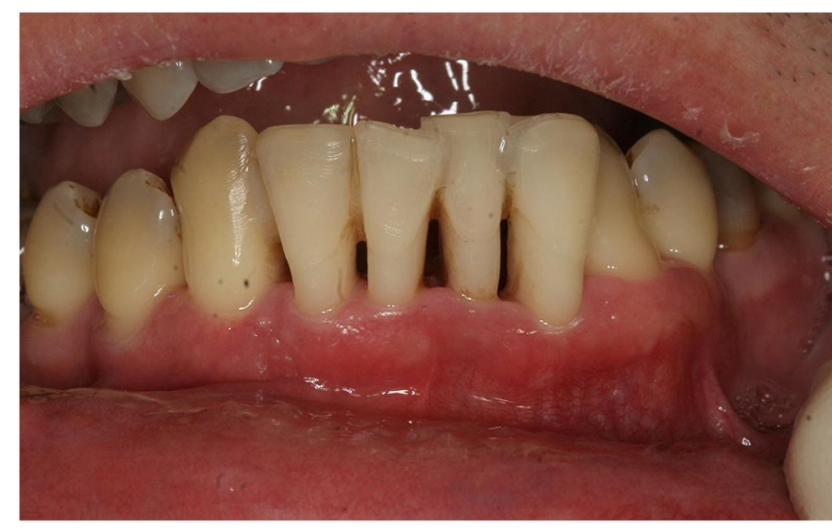

Figure 4 Intraoral view of the frontal lower teeth I4 days after SRP and splinting.

No pus discharge or bleeding on probing was observed at a 6-month follow-up appointment (Figure 5). Panoramic radiographs (Figure 6) revealed that the alveolar bone level was considered stable compared with previous radiographs. The bone distal from tooth \#34 healed, and tooth \#43 showed an extensive periapical lesion with well-defined borders.

Intact tooth \#43 was not responsive to the cold test. The electric pulp test confirmed that the pulp was nonvital. Vertical percussion was slightly tender when compared with the neighboring teeth. The alveolar mucosa had no fistulas or edema. The pocket depth of tooth \#43 was probed via the "walking" probing technique ${ }^{19}$ with the University of Michigan's “O” probe using Williams markings (at 1, 2, 3, 5, 7, 8, 9, and $10 \mathrm{~mm}$ ). All of the measurements were $2 \mathrm{~mm}$, except for the mesial side, where a narrow $8 \mathrm{~mm}$ periodontal pocket and $10 \mathrm{~mm}$ of clinical attachment loss was revealed (Figure 7A-D). The

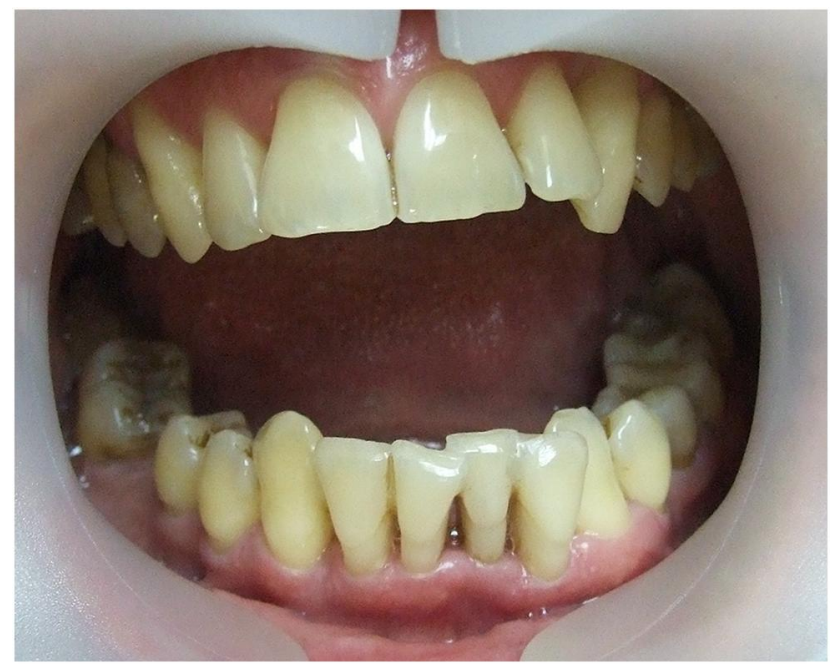

Figure 5 Intraoral view 6 months after SRP. 


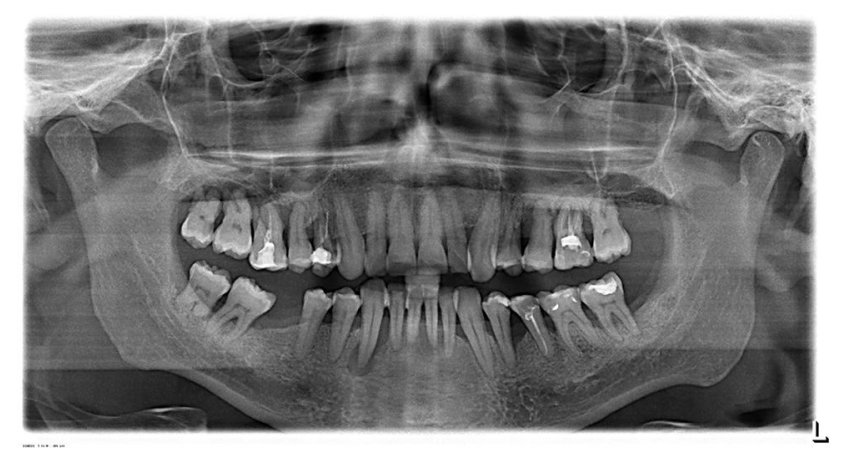

Figure 6 Panoramic radiograph 6 months after SRP: tooth \#43 showed an extensive periapical lesion with well-defined borders and bone loss extruding onto the root's mesial surface.

diagnosis was confirmed as follows: chronic apical periodontitis according to ICD- $10,{ }^{20}$ and primary periodontal disease with secondary endodontic involvement according to Rotstein and Simon's ${ }^{4}$ classification. As far as a narrow pocket may also be present in vertical root fracture, the differential diagnosis with vertical root fracture was done according to the criteria of Byakova et $a{ }^{21}$ and vertical root fracture was not revealed.

The patient was referred to an endodontist and underwent endodontic treatment (Figure 8A-D) according to the following protocol: administration of local anesthesia using $1.7 \mathrm{~mL}$ of $4 \%$ articaine (Ubistesin Forte, $3 \mathrm{M}$ ESPE), rubber dam isolation, and preparation of endodontic access. No bleeding occurred during the root canal treatment. Necrotic pulp was found, and the working length was confirmed with radiographs (Figure 9A). Root canal instrumentation was conducted with a rotary nickeltitanium instrument (iRace, FKG Dentarium). The root canal was irrigated with $3 \%$ sodium hypochlorite (Parcan, Septodont), distilled water, and 17\% EDTA (EndoGiNo. 2, 17\% EDTA, VladMiva). Sodium hypochlorite was activated using a passive ultrasound tip (Irrisafe IRR-2025, Satelec/Acteon). The root canal was dried and treated with ozone gas from an ozone generator (Prozone, W\&H) for 24 seconds following endodontic setting in Prozone (Figure 10).

The root canal was dressed with calcium hydroxide (Calasept, Nordiska Dental AB) for 7 days. After 1 week, the patient reported no pain or discomfort when biting in tooth \#43. The root canal was disinfected again using the same protocol. After drying, the root canal was obturated with lateral compaction using guttapercha points and sealer (AH Plus, Dentsply). Obturation was controlled with radiographs
A

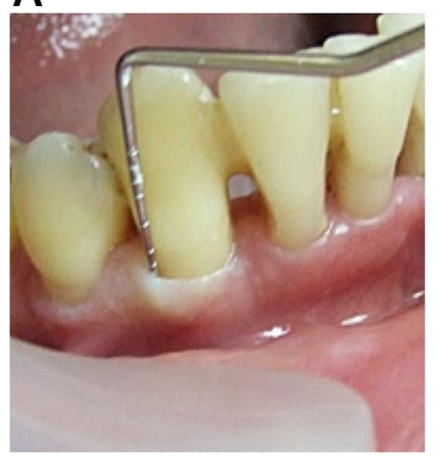

B

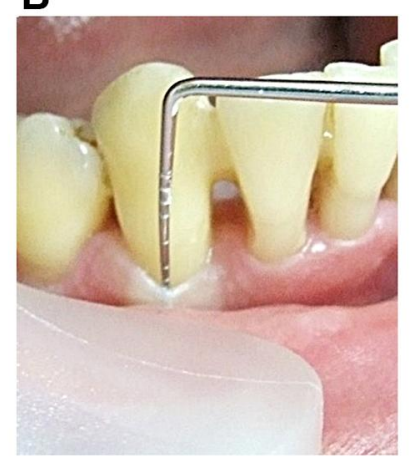

C

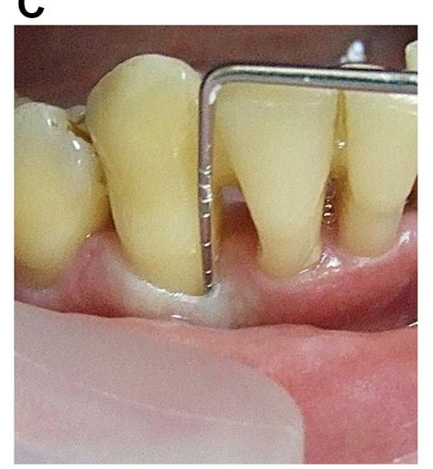

D

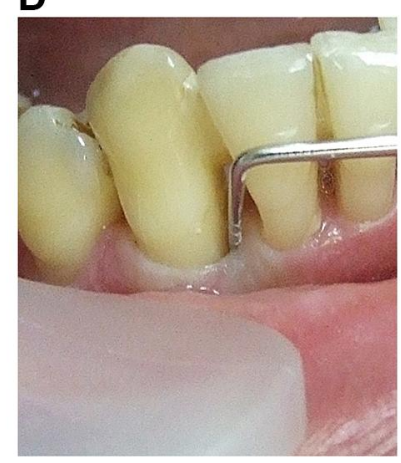

Figure 7 "Walking" probing of the periodontal pocket using a Naber periodontal probe. Distal buccal point (A), middle buccal point (B), mesial buccal point (C), all measurements are $2 \mathrm{~mm}$ probing depth. (D) Narrow and deep $8 \mathrm{~mm}$ periodontal pocket on the mesial tooth surface.

A

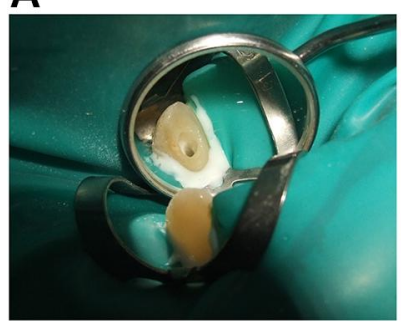

B

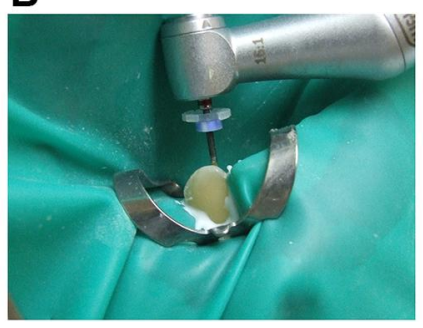

C

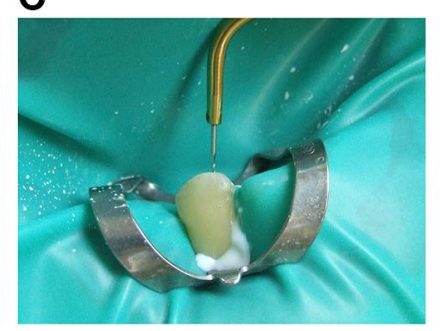

D

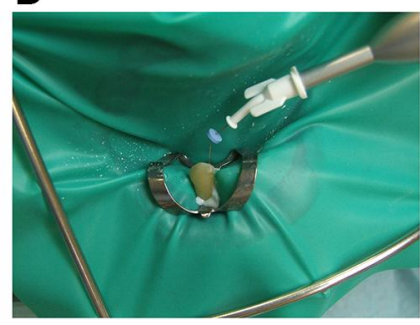

Figure 8 (A) No bleeding was observed immediately after endodontic access opening. (B) Instrumentation of the root canal using an iRace rotary endodontic instrument. (C) Passive ultrasound irrigation with an ultrasound tip. (D) Treating the root canal with ozone gas. 
A

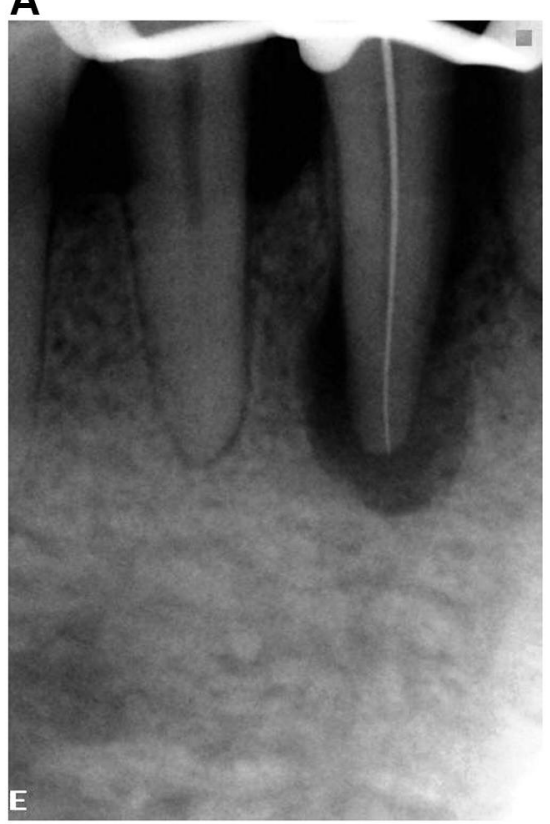

B

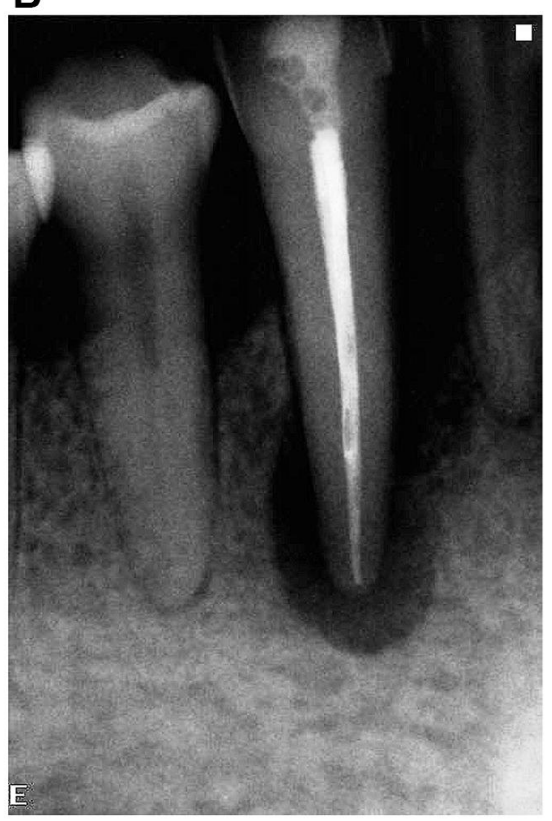

C

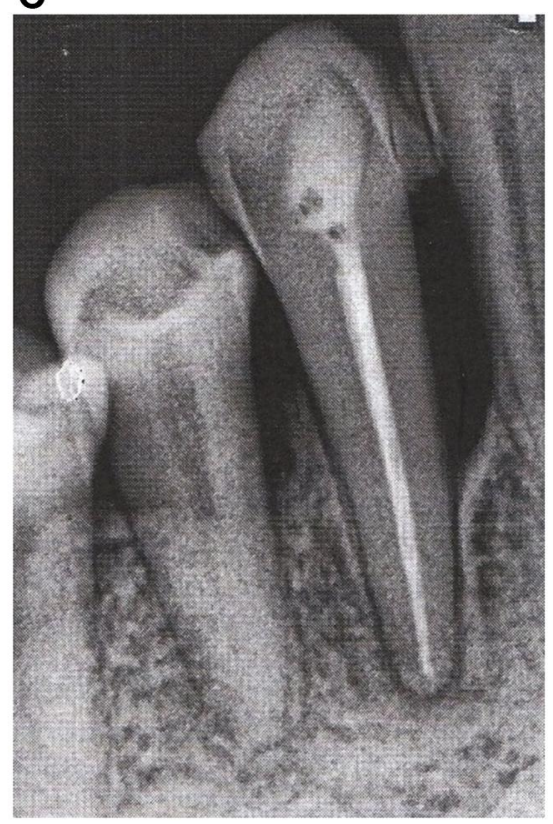

Figure 9 (A) Working length confirmation. (B) Immediately after obturation. (C) Six-month follow-up showing complete healing of the bone defect in the periapical area and interdental septum.

(Figure 9B). Endodontic access was filled with a light-cured composite (Revolution, Kerr and Filtek Z250, 3M ESPE).

The day after endodontic treatment, root scaling and planing were performed. The periodontal pockets were rinsed with chlorhexidine and treated with ozone gas for 18 seconds according to the device's periodontal treatment settings.

The patient returned for a follow-up appointment 6 months after endodontic treatment Periapical radiograph demonstrated complete healing of the periapical lesion and reorganization of the alveolar bone lateral to the tooth

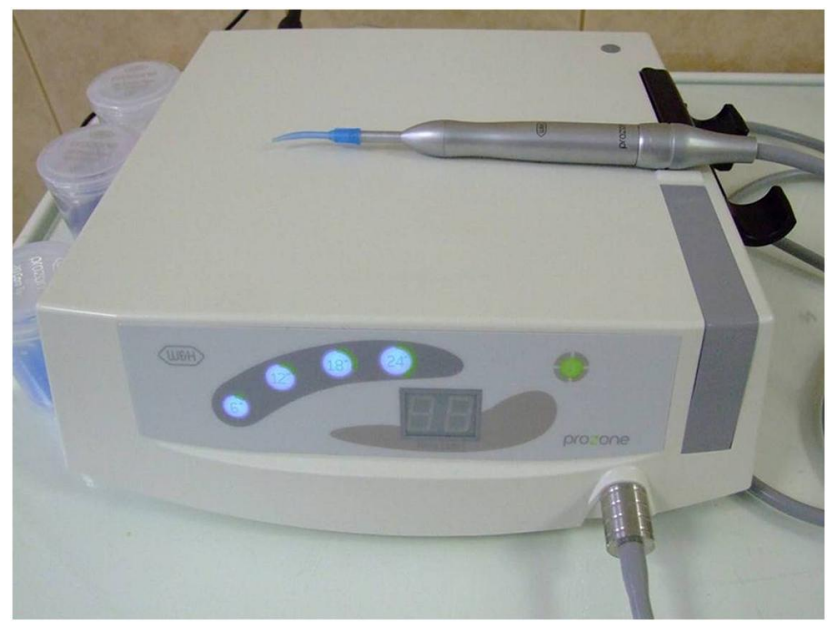

Figure 10 Prozone ozone generator. surface (Figure 9C). The probing depth mesial from tooth \#43 was $4 \mathrm{~mm}$.

\section{Discussion}

Endo-perio lesions present challenging diagnosis, differentiation, and treatment. ${ }^{22}$ Thorough clinical examinations and radiological investigations enable the definition of lesion's causative factors. While endodontic infection in periapical tissues may be verified with radiographs, probing the pocket depth and measuring the clinical attachment loss are essential for detailed evaluation of periodontal destruction. In the present case, the endo-perio lesion's periodontal pocket was very narrow. This finding was confirmed by Hind et $\mathrm{al}^{23}$ who also reported narrow periodontal pockets in endo-perio lesions. Because narrow periodontal pockets may be clinically missed, we used "walking" probing ${ }^{19}$ instead of conventional probing at 4-6 points. This technique is recommended to diagnose teeth with endo-perio lesions because it decreases the risk of missing narrow pockets.

A randomized clinical trial can generate high-quality evidence when evaluating an intervention's effectiveness.${ }^{24}$ Unfortunately, there is a lack of such studies on endoperio lesions because their pathology is relatively rare. ${ }^{4}$ Affected teeth often have poor prognosis, ${ }^{25}$ and the variety of clinical parameters are difficult to standardize. Thus, detailed reviews of clinical cases may be valuable for 
clinicians to define treatment strategies and anticipate particular clinical outcomes.

The latest review of endo-perio lesions clinical cases was published in 2009 by Oh et al. ${ }^{26}$ Twenty-six cases from 14 articles were evaluated. The authors proposed a 4phase treatment algorithm: 1) presurgical phase (determining periodontal/regenerative prognosis), 2) endodontic phase, 3) periodontal surgical phase, and 4) post-GTR reevaluation protocol. This might provide beneficial guidelines for managing endodontic-periodontal combined lesions.

Although a general treatment strategy was summarized, the sequence and details of treatment remain controversial. Gupta et $\mathrm{al}^{27}$ reported that non-surgical periodontal treatment may be conducted simultaneously with endodontic treatment for the management of concurrent endodontic-periodontal lesions without communication. Schmidt et $\mathrm{al}^{28}$ postulated that a sequential strategy with root canal treatment as a first step is reasonable. Abbott ${ }^{29}$ concluded that endodontic treatment can be completed before periodontal treatment, except where there is a "combined endodontic-periodontal lesion with communication". In these cases, root canals should be medicated until periodontal treatment has been completed. There are literature data about successful treatment of endoperio lesions using platelet-rich fibrin and plasma for treatment of combined endo-perio lesions. ${ }^{30}$

The absence of a universal international classification of endo-perio lesions makes comparing publications problematic because of differences in terminology. Regarding the diversity of recommendations and terminology and the lack of randomized clinical trials, cases and case series reviews with detailed presentations of baseline clinical and radiological parameters may be beneficial for clinicians, allowing them to compare baseline parameters, treatment strategies, and clinical outcomes with endo-perio lesion cases in their practise.

This review included case studies from the PubMed database on March 2, 2020, using the search terms "endo perio lesion", "periodontic-endodontic lesion", and "endodontic-periodontic lesion" from 2011 to 2019.There were 29 cases from 17 case reports and case series. The following parameters were evaluated: patient age and systemic health, tooth, lesion type according to Rotstein and Simon's ${ }^{4}$ classification, baseline parameters (teeth vitality, radiograph [RVG], probing depth, tooth mobility, and furcation involvement), splinting, treatment protocol, and degree of healing at follow-up appointments. The present case was compared with similar cases from the review. All the obtained data from the review were organized into tables according to the lesion type (Tables 1-4).

In the review, most of the patients had no systemic conditions; one patient had diabetes mellitus. ${ }^{31}$ Healing in this patient was slower compared with healthy patients ${ }^{32}$ with the same lesion type, mobility, and probing depth in single-rooted teeth. An 18-month follow-up RVG of the diabetic patient showed decreased radiolucency and a 39month follow-up RVG showed complete healing of the lesion, while, in healthy patients, a 14-month RVG showed resolution of bone defects. Faster healing in healthy patients may be explained by the absence of systemic pathology, immediate splinting (while in the diabetic patient, splinting was delayed for 6 months after endodontic treatment), and regenerative periodontal surgery 3 months after endodontic treatment (the diabetic patient underwent open flap surgery without grafting 6 months after endodontic treatment). In systemically compromised patients, endo-perio lesions represent higher risks because focal dental infections have negative effects on systemic conditions $^{33}$ and may trigger exacerbation of some autoimmune pathologies such as psoriasis and rheumatoid arthritis. $^{34,35}$ Regular follow-ups for healing control are essential to prove treatment success and prevent focal dental infections.

There were 12 teeth (six molars ${ }^{16,23,36,37}$ and six single-rooted teeth ${ }^{23,36,37}$ ) with PESP in the review. All molars had furcation involvement. Four molar cases did not undergo periodontal regenerative surgery and showed decreased radiolucency in 12-month RVG. ${ }^{36}$ Two molar cases underwent regenerative surgery. One showed complete healing in 6-month $\mathrm{RVG}^{37}$ and the other demonstrated complete healing in 2-year RVG with no information about the 6-month follow-up. ${ }^{16}$

Four single-rooted teeth with PESP underwent endodontic treatment and SRP without regenerative procedures. $^{36}$ Two teeth received endodontic treatment and SRP with regenerative procedures. ${ }^{23,38}$ None of the teeth without regeneration surgery showed complete healing of bone defects at a 6-month follow-up RVG. One tooth with regeneration surgery demonstrated complete healing of bone defects at a 6-month follow-up RVG. ${ }^{38}$ Regenerative surgery seems to accelerate healing in molars and single-rooted teeth with PESP. This conclusion should be confirmed by future clinical studies.

There were 18 teeth with PPSE (six molars ${ }^{10,-16,-39-41}$ and 12 single-rooted teeth ${ }^{35,42-46}$ ) in the reviewed literature. Two molars were extracted without any treatment. ${ }^{40}$ Two 


\begin{tabular}{|c|c|c|c|c|c|c|c|}
\hline 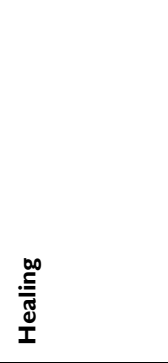 & 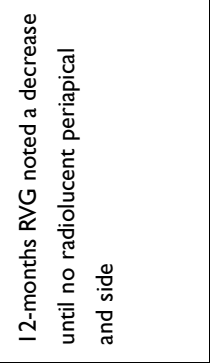 & 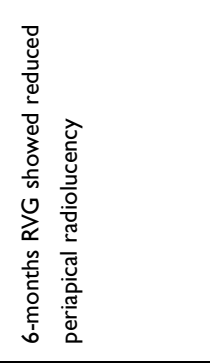 & 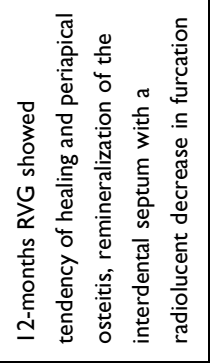 & 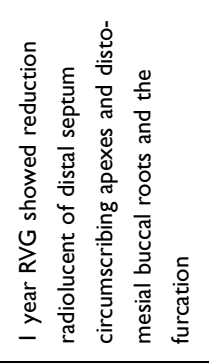 & 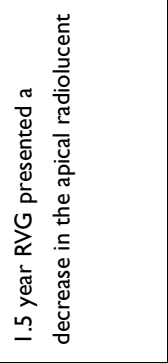 & 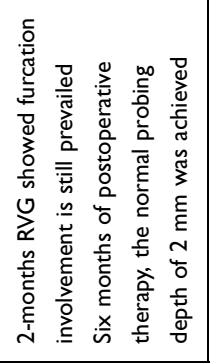 & 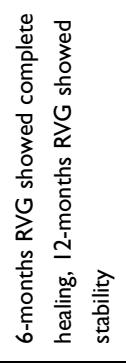 \\
\hline 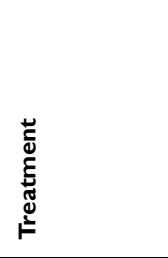 & 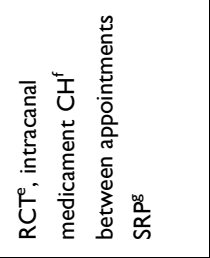 & 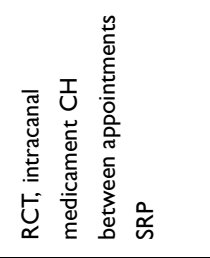 & 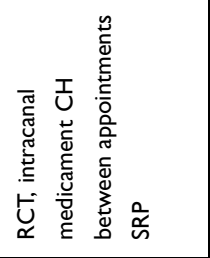 & 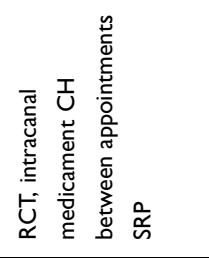 & 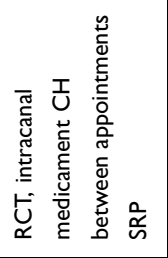 & 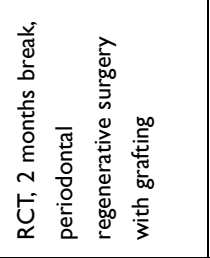 & 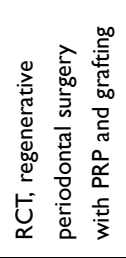 \\
\hline 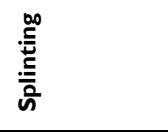 & $\stackrel{0}{z}$ & $\stackrel{0}{z}$ & $\stackrel{\circ}{z}$ & $\stackrel{0}{z}$ & $\stackrel{\circ}{z}$ & $\stackrel{0}{z}$ & $\stackrel{0}{z}$ \\
\hline 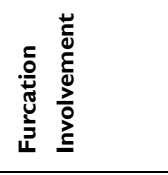 & 1 & 1 & 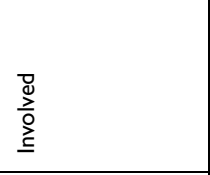 & 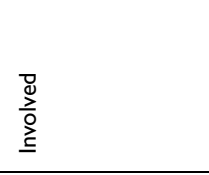 & 1 & 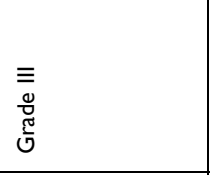 & \\
\hline 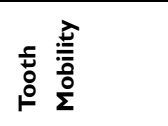 & 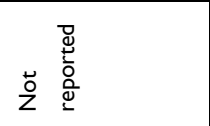 & z) & 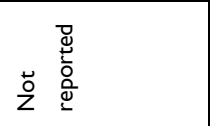 & 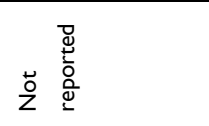 & 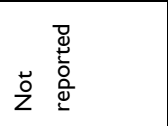 & 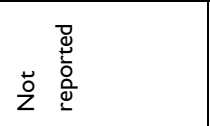 & 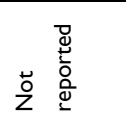 \\
\hline ă & $\begin{array}{l}E \\
\xi \\
\stackrel{E}{+} \\
\end{array}$ & 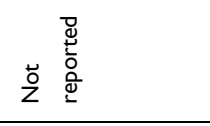 & $\begin{array}{l}\varepsilon \\
\varepsilon \\
\\
\end{array}$ & 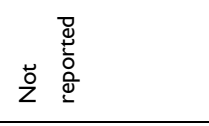 & 苋 & $\begin{array}{l}\varepsilon \\
\underline{\varepsilon} \\
\stackrel{\varrho}{\underline{O}} \\
\end{array}$ & $\begin{array}{l}E \\
\xi \\
\infty \\
\end{array}$ \\
\hline 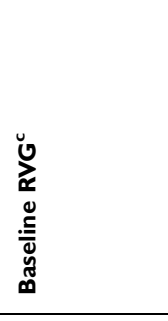 & 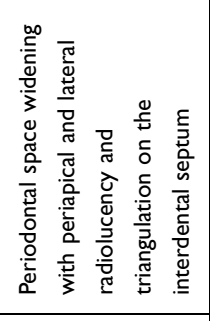 & 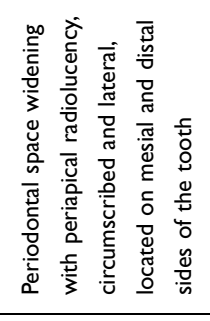 & 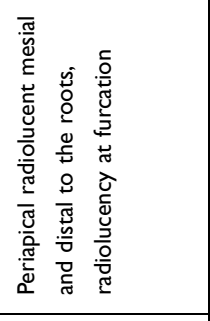 & 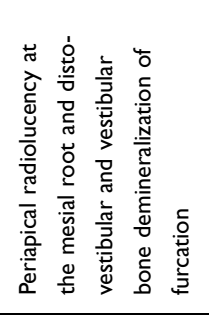 & 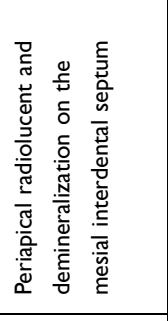 & 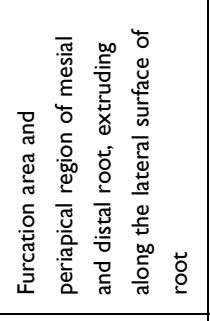 & 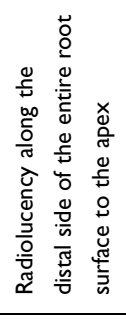 \\
\hline 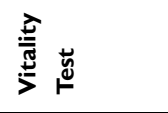 & 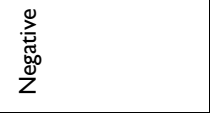 & 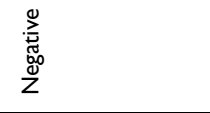 & 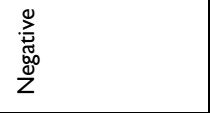 & 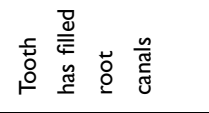 & 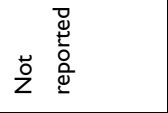 & 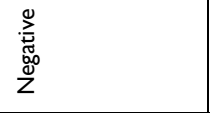 & 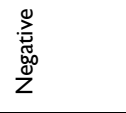 \\
\hline 它 & 总 & 总 & 畜 & 畜 & 总 & 总 & 离 \\
\hline $\begin{array}{l}\stackrel{5}{\circ} \\
\stackrel{\circ}{\circ} \\
\end{array}$ & $\underset{\#}{\#}$ & 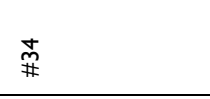 & 䒠 & 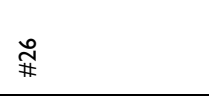 & $\overline{\overline{\#}} \bar{N}$ & 弯 & $\underset{\#}{\#}$ \\
\hline 品 & $\stackrel{\infty}{\infty}$ & $\stackrel{\infty}{\circledR}$ & \% & $\stackrel{\text { n }}{m}$ & $\stackrel{\sim}{\sim}$ & $\approx$ & 只 \\
\hline 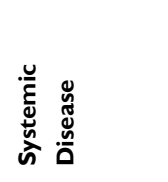 & 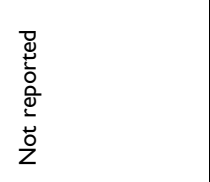 & 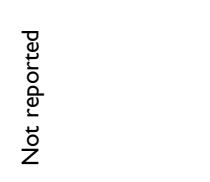 & 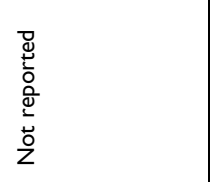 & 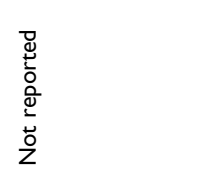 & 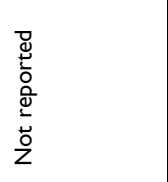 & $\begin{array}{l}\frac{\widehat{T}}{\frac{F}{J}} \\
\frac{\mathbb{\Phi}}{I}\end{array}$ & \\
\hline 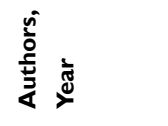 & \multicolumn{5}{|l|}{ 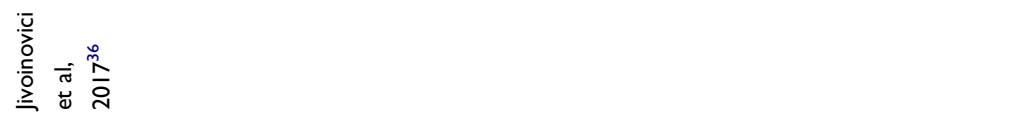 } & 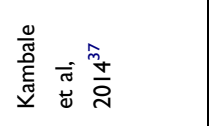 & 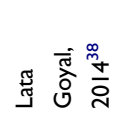 \\
\hline
\end{tabular}




\begin{tabular}{|c|c|c|c|c|}
\hline 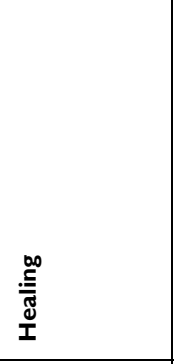 & 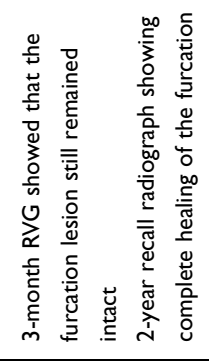 & 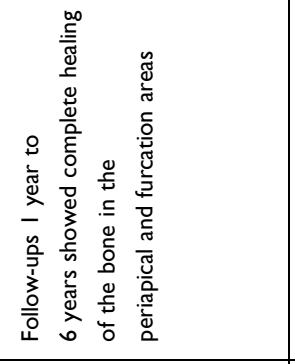 & 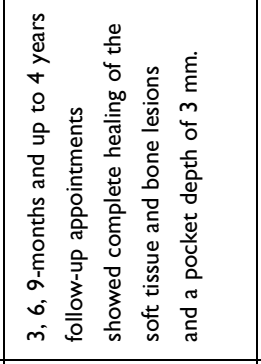 & 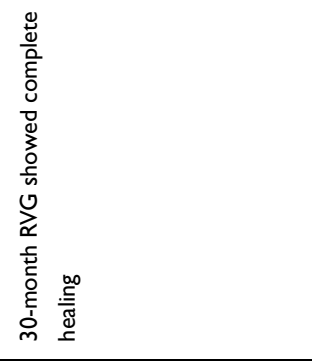 \\
\hline 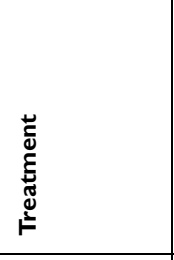 & 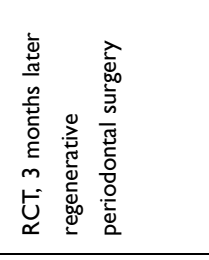 & 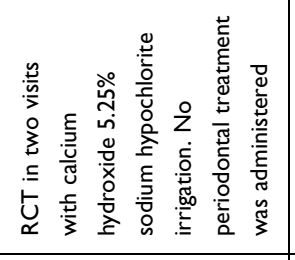 & 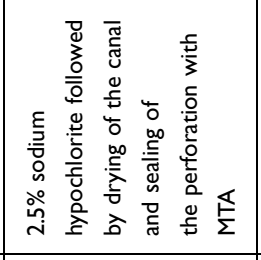 & 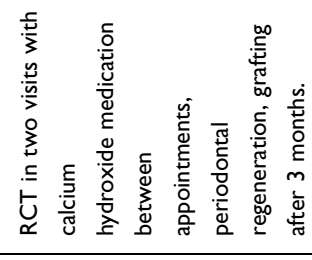 \\
\hline 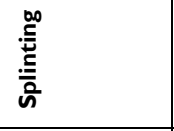 & $\stackrel{0}{z}$ & $\stackrel{0}{z}$ & $\stackrel{0}{z}$ & $\stackrel{0}{2}$ \\
\hline 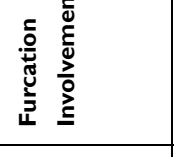 & 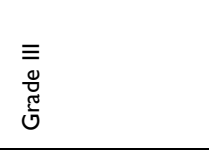 & 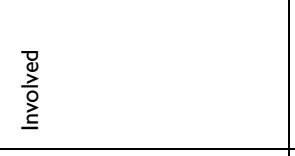 & \begin{tabular}{|l}
$\overline{\overline{0}}$ \\
$\overline{\tilde{e}}$ \\
$\overline{\tilde{O}}$ \\
\end{tabular} & 1 \\
\hline 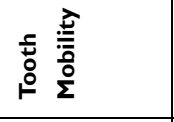 & $\check{\check{\sigma}}$ & 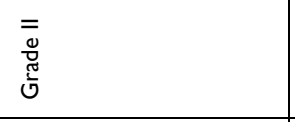 & 总 & $\begin{array}{l}\overline{\overline{0}} \\
\text { 尊 } \\
\end{array}$ \\
\hline$\stackrel{0}{0}$ & $\begin{array}{l}\underline{E} \\
\simeq \\
\end{array}$ & 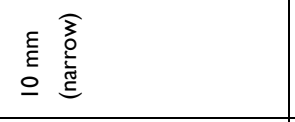 & 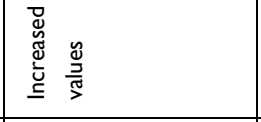 & $\begin{array}{l}\underline{\underline{\underline{E}}} \\
\underline{\underline{a}} \\
\end{array}$ \\
\hline 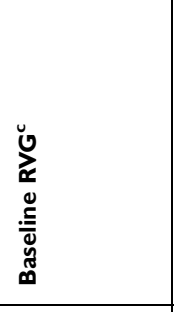 & 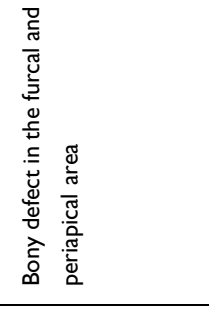 & 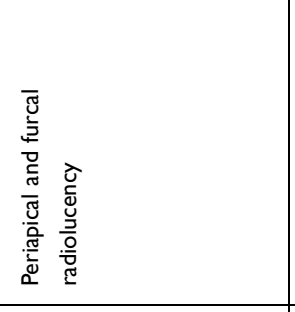 & 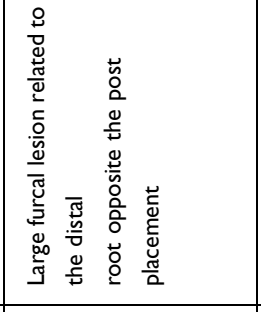 & 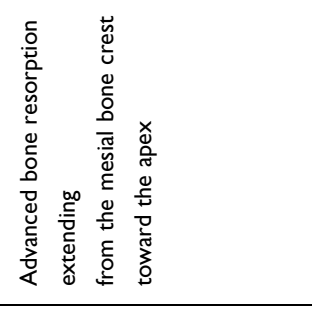 \\
\hline 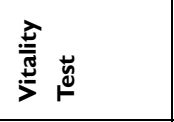 & 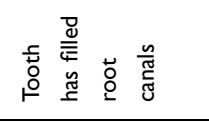 & 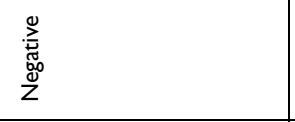 & 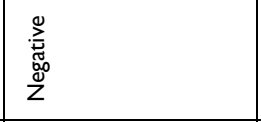 & 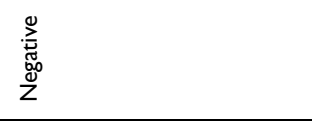 \\
\hline 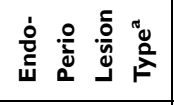 & 竞 & 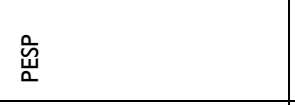 & \begin{tabular}{|l} 
产 \\
a.
\end{tabular} & 善 \\
\hline 畧 & $\dddot{\wp}$ & 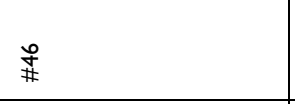 & ஐ & ¥ \\
\hline$\underset{8}{\stackrel{\circ}{<}}$ & ช & ڤొ & \% & $\lambda$ \\
\hline 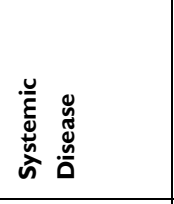 & \begin{tabular}{|l} 
琂 \\
总 \\
\end{tabular} & 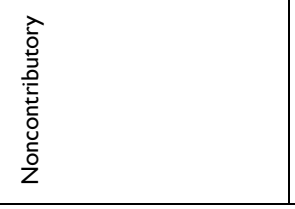 & 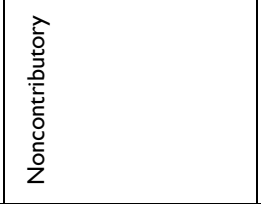 & 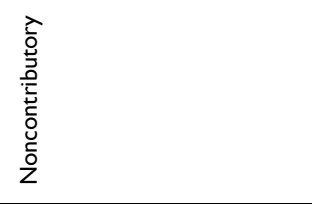 \\
\hline 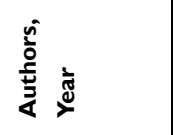 & 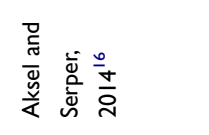 & \multicolumn{3}{|l|}{ 豆 } \\
\hline
\end{tabular}




\begin{tabular}{|c|c|c|c|c|c|c|}
\hline 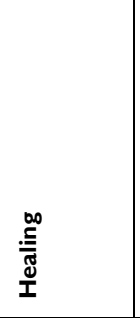 & 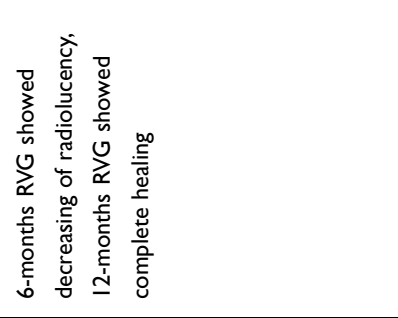 & 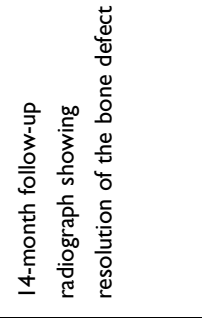 & 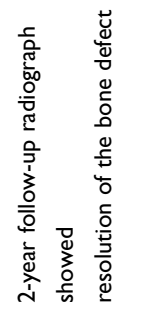 & 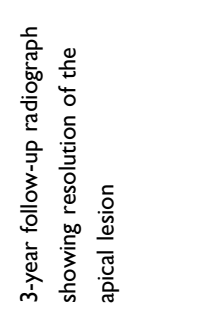 & 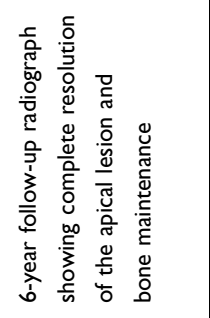 & 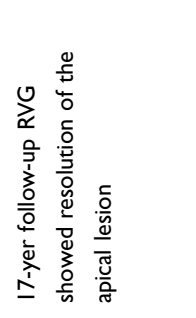 \\
\hline 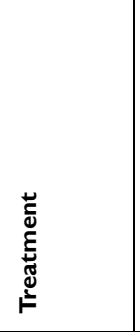 & 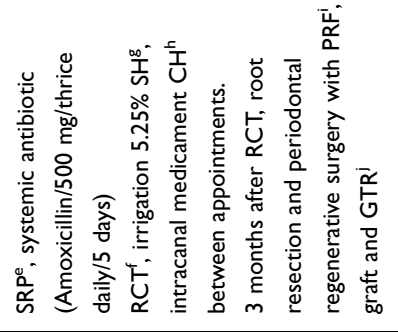 & 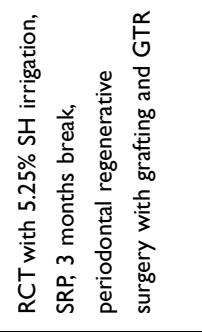 & 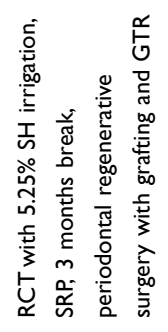 & 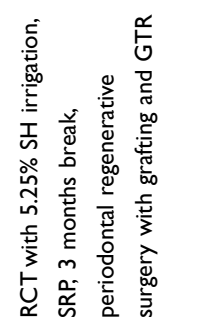 & 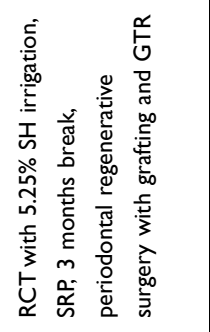 & 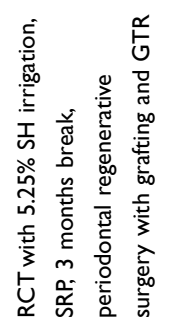 \\
\hline 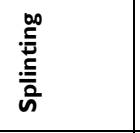 & z & $\stackrel{\Xi}{\check{g}}$ & $\underbrace{\tilde{u}}$ & $\stackrel{\Xi}{\check{\sigma}}$ & $\stackrel{0}{z}$ & ż \\
\hline 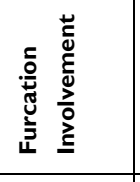 & 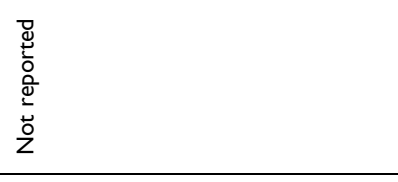 & 1 & 1 & 1 & 1 & 1 \\
\hline 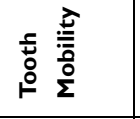 & 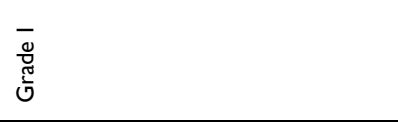 & 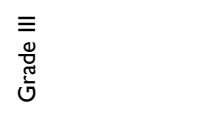 & 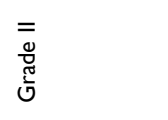 & 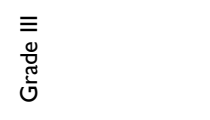 & $\begin{array}{l}\overline{\overline{0}} \\
\frac{\tilde{0}}{\tilde{0}} \\
\frac{\pi}{0}\end{array}$ & 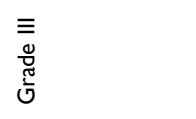 \\
\hline ō & $\begin{array}{l}\varepsilon \\
\xi \\
\sigma \\
\end{array}$ & $\begin{array}{l}\varepsilon \\
\Xi \\
\simeq \\
\end{array}$ & $\begin{array}{l}E \\
E \\
\underline{m} \\
\end{array}$ & 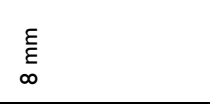 & $\begin{array}{l}\varepsilon \\
\varepsilon \\
\sigma \\
\end{array}$ & $\begin{array}{l}\varepsilon \\
\underline{\varepsilon} \\
\underline{O} \\
\end{array}$ \\
\hline 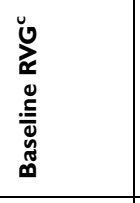 & 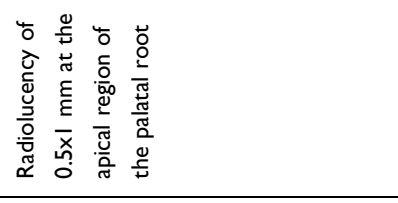 & 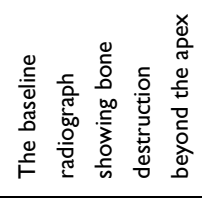 & 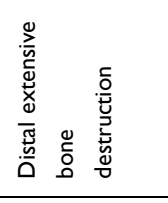 & 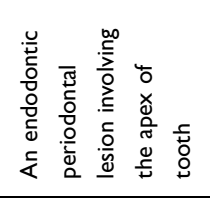 & 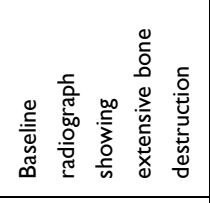 & 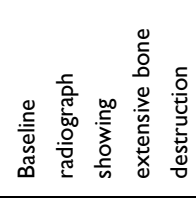 \\
\hline 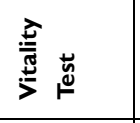 & 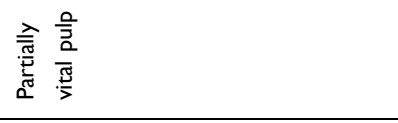 & 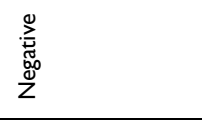 & 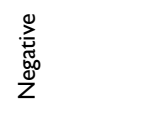 & 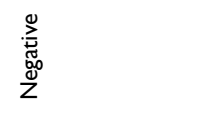 & 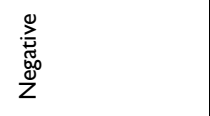 & 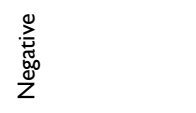 \\
\hline 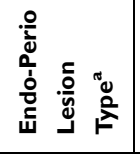 & 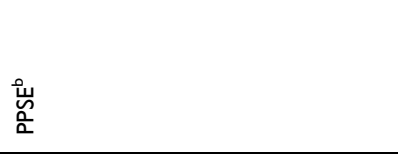 & 㟥 & 㟥 & 訔 & 訔 & 亳 \\
\hline $\begin{array}{l}\stackrel{f}{\circ} \\
\stackrel{\circ}{\circ}\end{array}$ & $\stackrel{\circ}{\#}$ & $\underset{\#}{\cong}$ & $\bar{\sharp}$ & $\bar{\sharp}$ & $\begin{array}{l}\overline{\text { s }} \\
\overline{\overline{\#}}\end{array}$ & $\overline{\overline{\#}}$ \\
\hline$\underset{8}{8}$ & q & 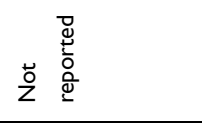 & 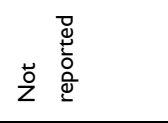 & 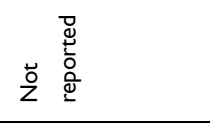 & 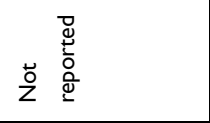 & 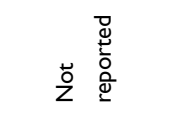 \\
\hline 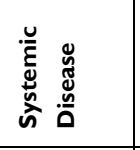 & $\begin{array}{l}\frac{\vec{F}}{\tilde{J}} \\
\frac{\tilde{J}}{I}\end{array}$ & 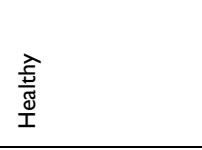 & 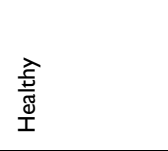 & 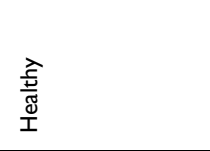 & 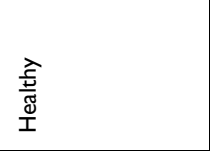 & 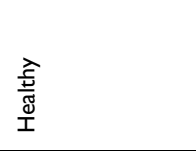 \\
\hline 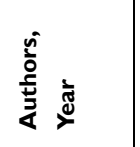 & 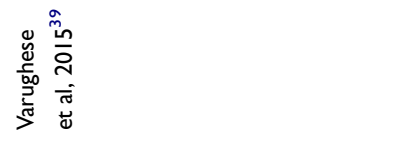 & \multicolumn{5}{|l|}{ 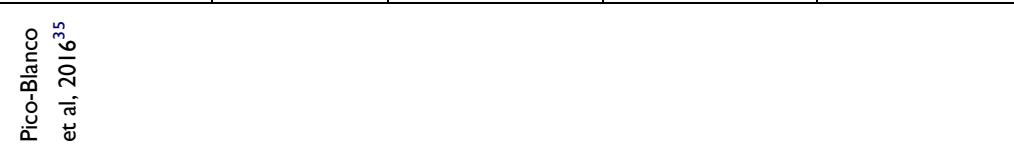 } \\
\hline
\end{tabular}




\begin{tabular}{|c|c|c|c|c|}
\hline $\begin{array}{l}\frac{\infty}{\stackrel{\infty}{\overline{0}}} \\
\frac{\tilde{\Phi}}{I}\end{array}$ & 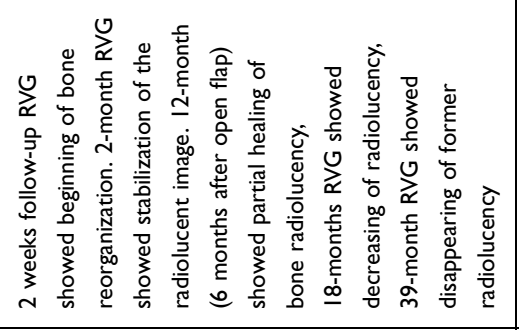 & 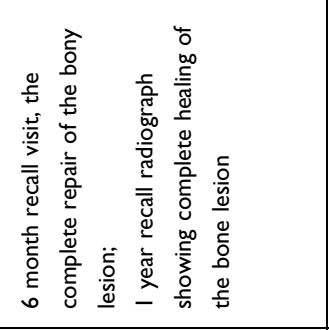 & . & 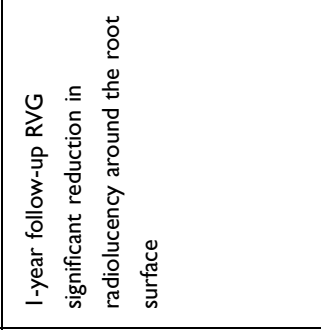 \\
\hline 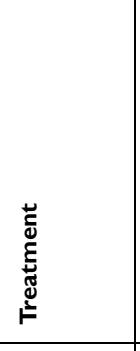 & 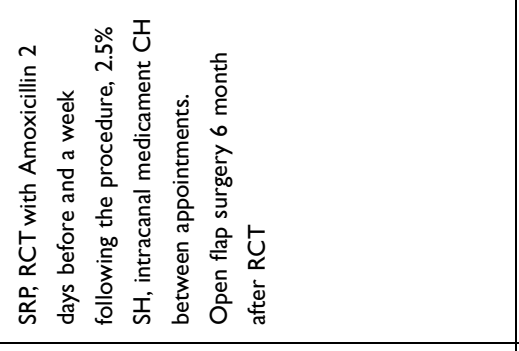 & 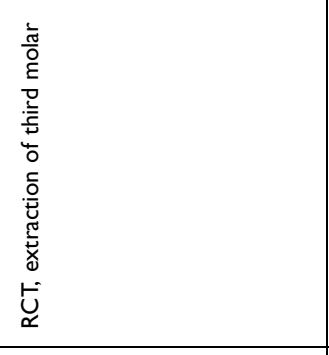 & 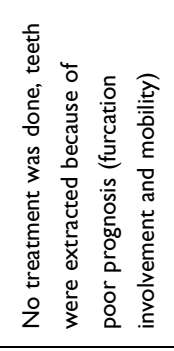 & 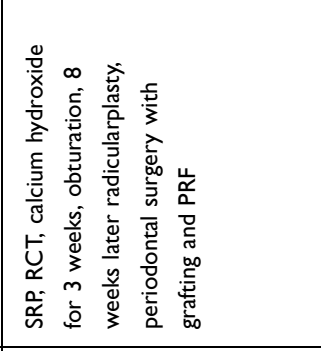 \\
\hline 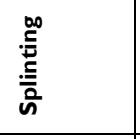 & 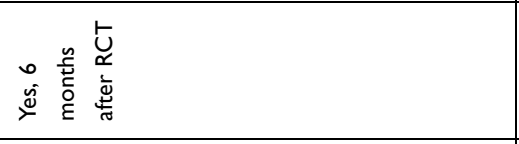 & \& & $\stackrel{0}{2}$ & z̊ \\
\hline 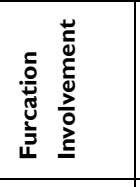 & . & 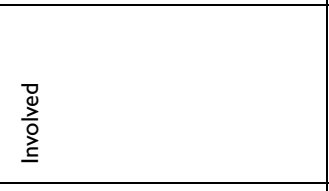 & \begin{tabular}{|l}
$\overline{\overline{1}}$ \\
$\overline{0}$ \\
$\overline{0}$ \\
$\overline{0}$ \\
\end{tabular} & . \\
\hline 亦高 & 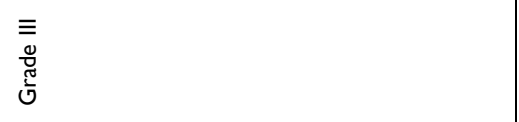 & 雚 & $\stackrel{0}{0}$ & 总 \\
\hline ŏ & $\begin{array}{l}\underset{\underline{E}}{\underline{E}} \\
\end{array}$ & 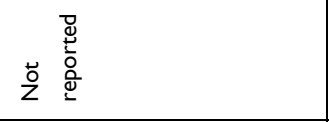 & 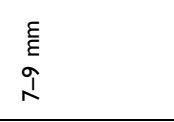 & $\begin{array}{c}\stackrel{\varepsilon}{\underline{\underline{\epsilon}}} \\
\underline{\underline{n}}\end{array}$ \\
\hline 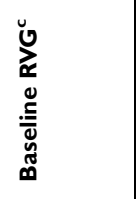 & 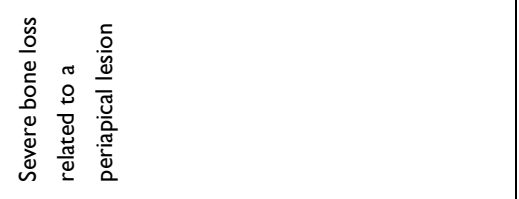 & 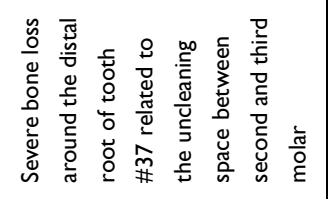 & 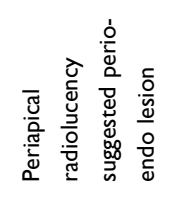 & 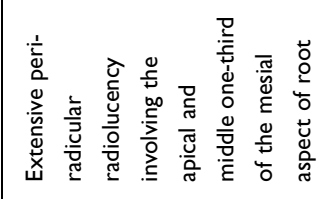 \\
\hline 逽 & 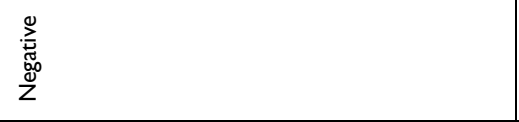 & 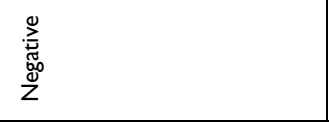 & 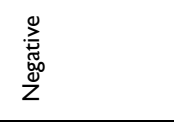 & 总 \\
\hline 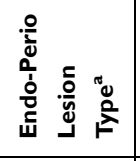 & 烹 & 訔 & 蕙 & 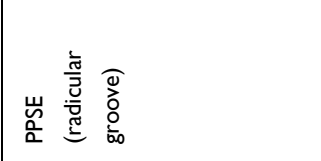 \\
\hline $\begin{array}{l}\stackrel{5}{\circ} \\
\stackrel{\circ}{\circ} \\
\end{array}$ & $\frac{m}{\#}$ & $\hat{\dddot{\#}}$ & $\frac{\dot{0}}{\#}$ ה & $\overline{\overline{\#}}$ \\
\hline$\underset{\&}{\&}$ & in & ₹ & 8 & $\stackrel{\sim}{m}$ \\
\hline 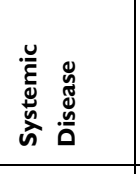 & 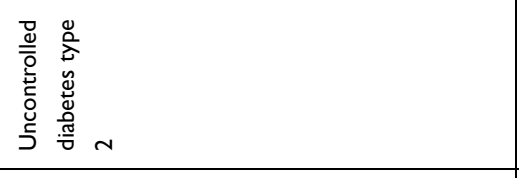 & 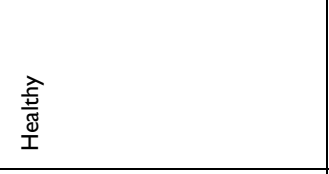 & 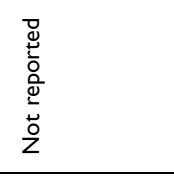 & 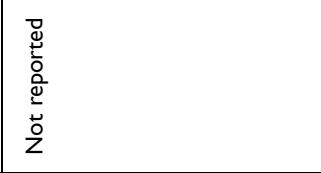 \\
\hline 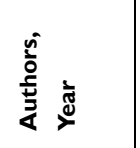 & 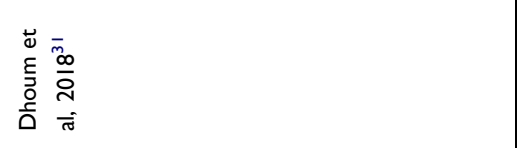 & 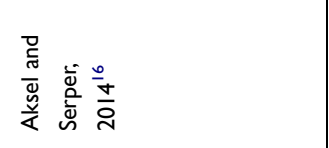 & 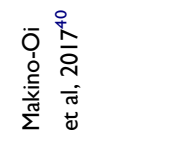 & 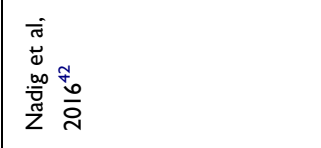 \\
\hline
\end{tabular}




\begin{tabular}{|c|c|c|}
\hline 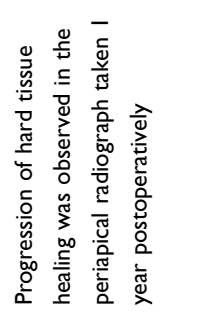 & 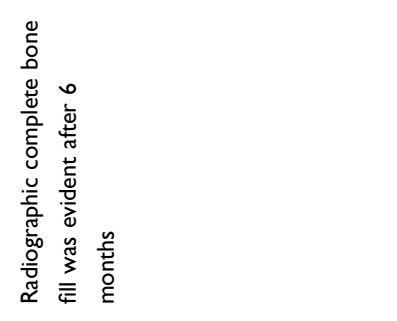 & 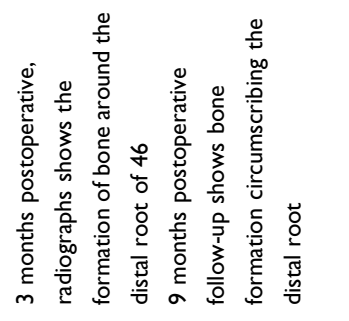 \\
\hline 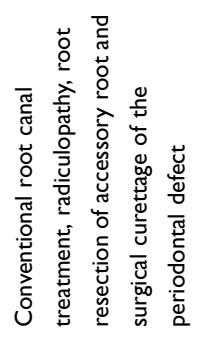 & 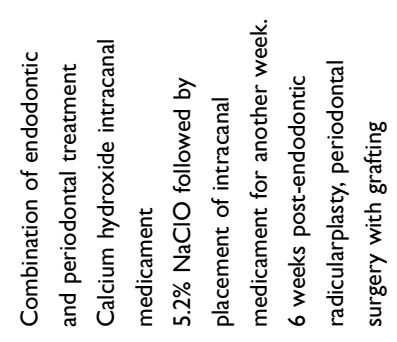 & 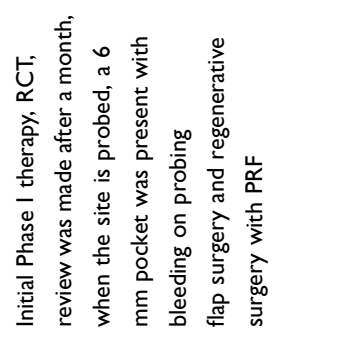 \\
\hline$\stackrel{\circ}{z}$ & zo & $\stackrel{0}{z}$ \\
\hline , & ' & 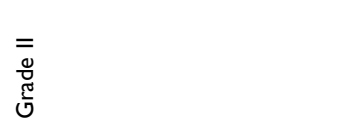 \\
\hline$\stackrel{\mathscr{\varpi}}{\tau}$ & $\stackrel{\check{\Xi}}{\tau}$ & 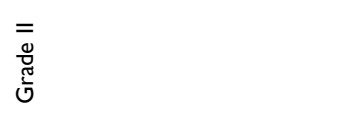 \\
\hline $\begin{array}{l}\underline{E} \\
\underline{\varepsilon} \\
\underline{\varrho}\end{array}$ & $\begin{array}{l}\underline{\varepsilon} \\
\underline{\underline{\varepsilon}}\end{array}$ & $\underset{\infty}{\underline{E}}$ \\
\hline 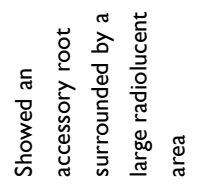 & 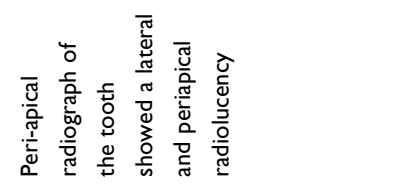 & 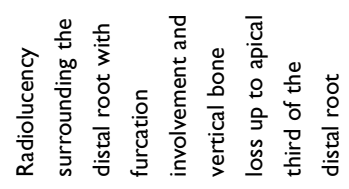 \\
\hline 惫 & 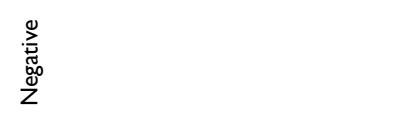 & 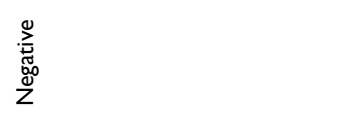 \\
\hline 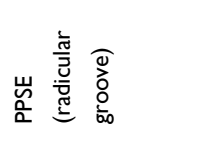 & 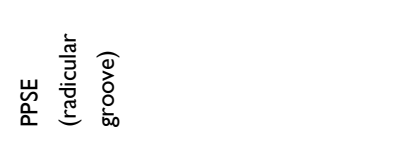 & 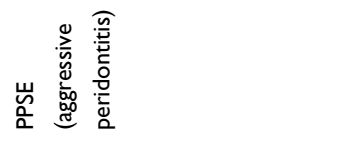 \\
\hline$\underset{\#}{\mathbb{\#}}$ & $\underset{\#}{\mathbb{\#}}$ & ¥ \\
\hline 요 & $\underline{\sigma}$ & $\stackrel{\infty}{\sim}$ \\
\hline 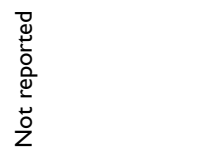 & 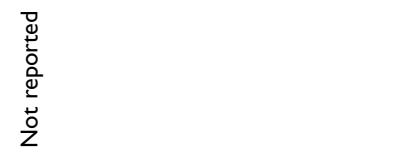 & $\begin{array}{l}\text { 訔 } \\
\text { 总 }\end{array}$ \\
\hline 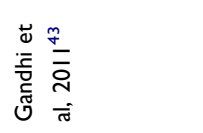 & 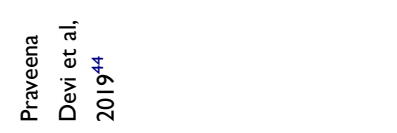 & 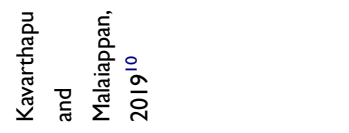 \\
\hline
\end{tabular}




\begin{tabular}{|c|c|c|c|}
\hline 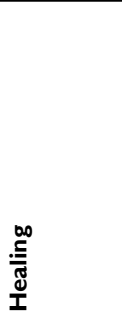 & 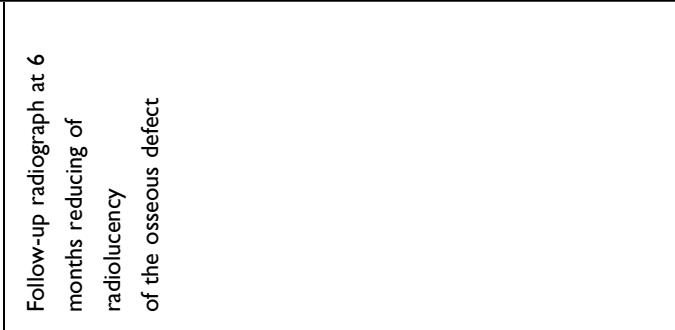 & 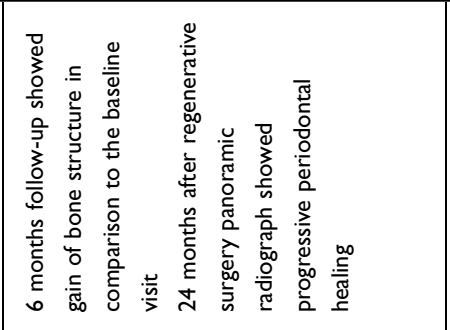 & 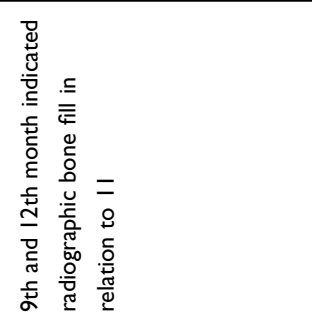 \\
\hline 䓂 & 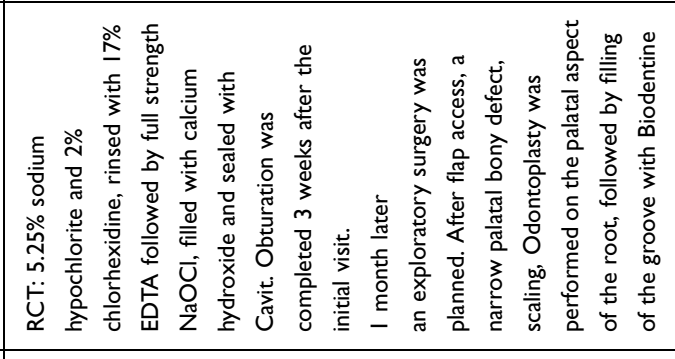 & 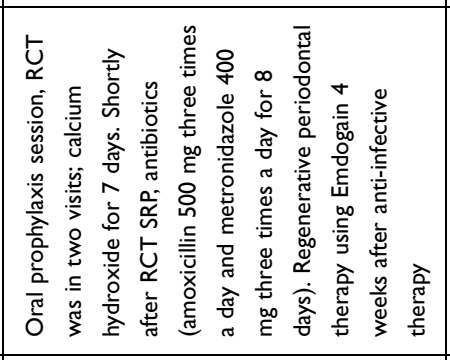 & 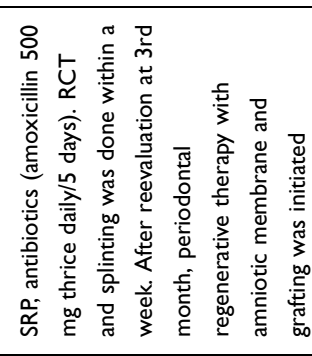 \\
\hline 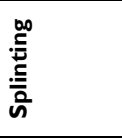 & z̊ & zo & 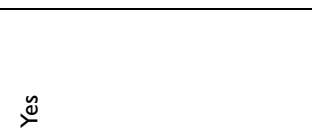 \\
\hline 䓂 & . & \begin{tabular}{|l}
$\overline{\mathrm{v}}$ \\
$\overline{\tilde{g}}$ \\
$\overline{\tilde{c}}$
\end{tabular} & , \\
\hline 吾 & 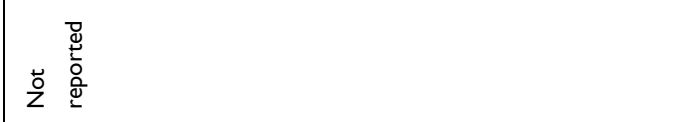 & 总 & $\begin{array}{l}\overline{\overline{0}} \\
\overline{8} \\
\frac{8}{5}\end{array}$ \\
\hline$\stackrel{\circ}{\circ}$ & 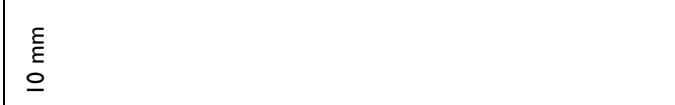 & 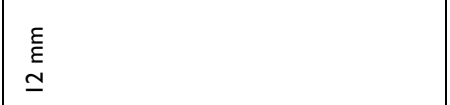 & 高 \\
\hline & 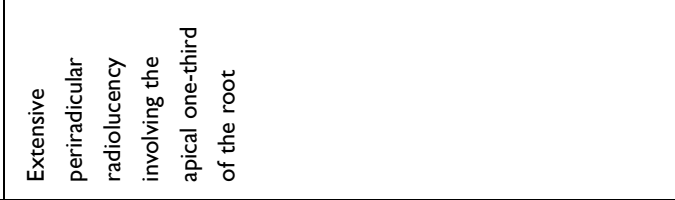 & 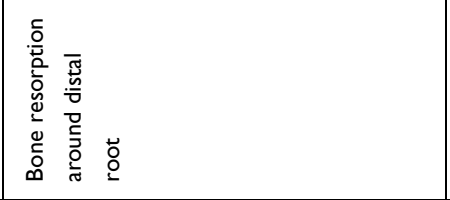 & 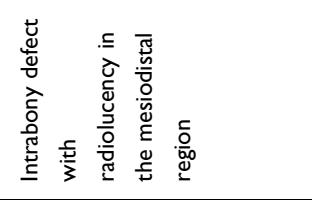 \\
\hline 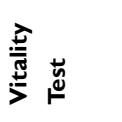 & 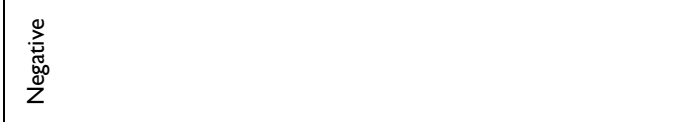 & 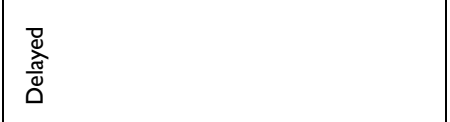 & $\overline{\mathrm{g}}$ \\
\hline هُ & 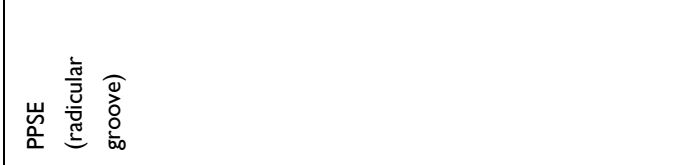 & 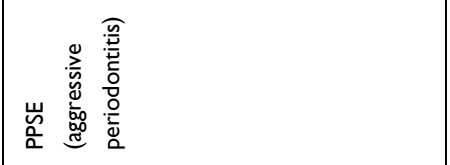 & 亳 \\
\hline 咅 & \# & 呆 & $\overline{\overline{\#}}$ \\
\hline 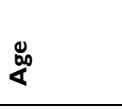 & I & $\stackrel{m}{m}$ & $\dot{m}$ \\
\hline 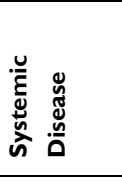 & 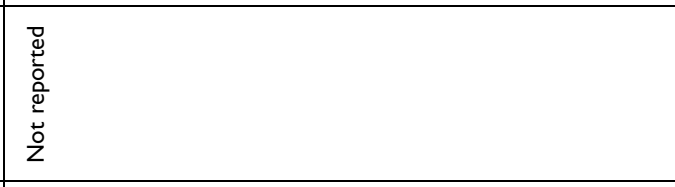 & 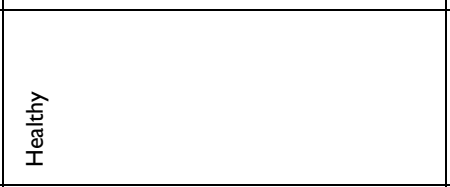 & 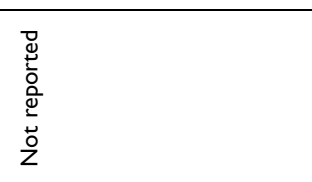 \\
\hline 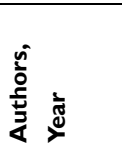 & 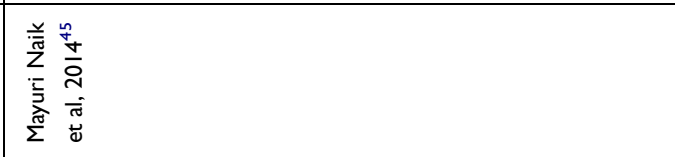 & 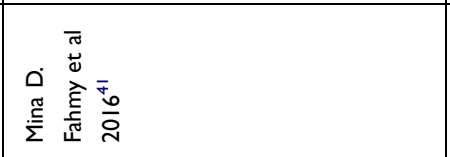 & 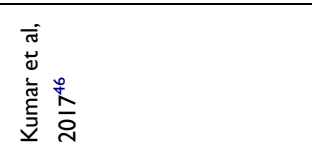 \\
\hline
\end{tabular}




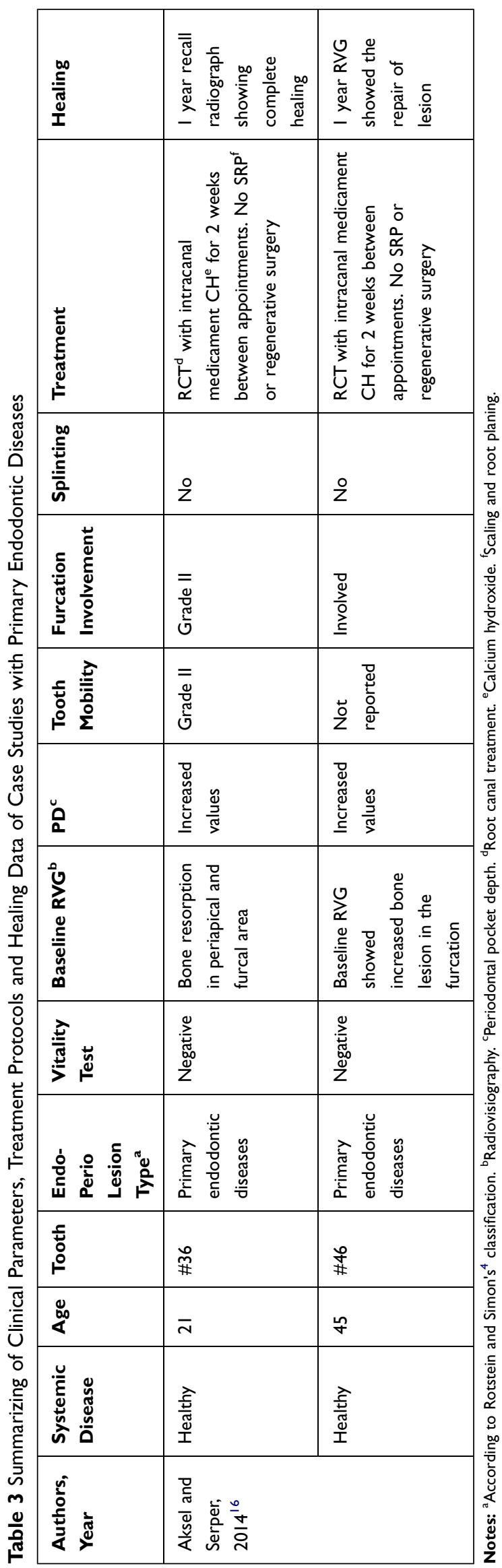

\begin{tabular}{|c|c|}
\hline 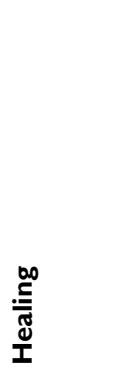 & 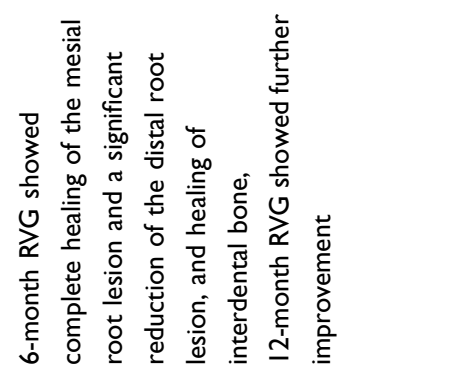 \\
\hline 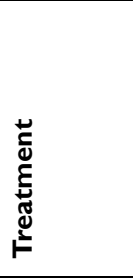 & 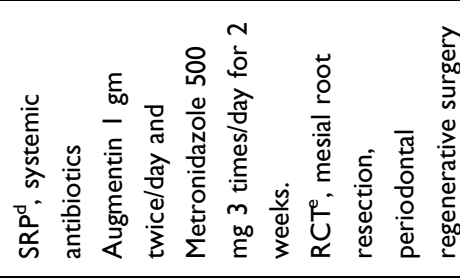 \\
\hline no & 우 \\
\hline 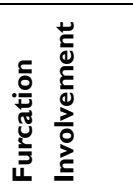 & $\begin{array}{l}\bar{\nabla} \\
\overline{\tilde{\sigma}} \\
\frac{\tilde{J}}{0}\end{array}$ \\
\hline 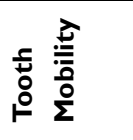 & $\begin{array}{l}\overline{0} \\
\overline{\tilde{\sigma}} \\
\overline{\tilde{0}}\end{array}$ \\
\hline 高 & 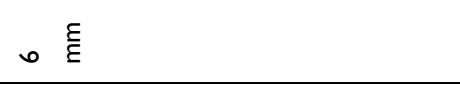 \\
\hline 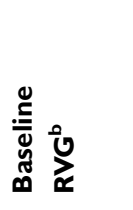 & 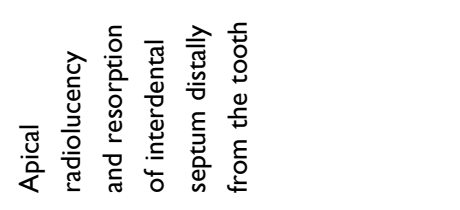 \\
\hline 氡 & 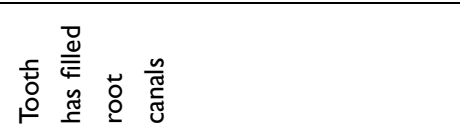 \\
\hline 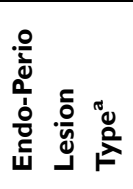 & 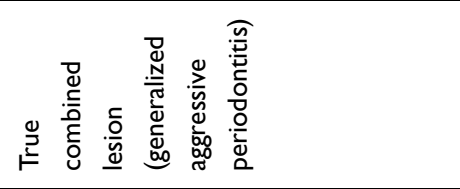 \\
\hline $\begin{array}{l}\text { 형 } \\
\stackrel{\circ}{\circ}\end{array}$ & ஐ \\
\hline$\stackrel{8}{8}$ & $\bar{m}$ \\
\hline 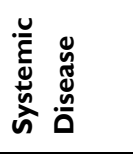 & 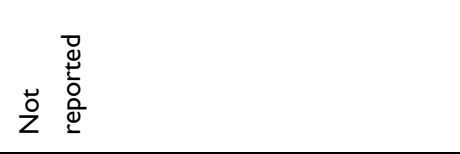 \\
\hline 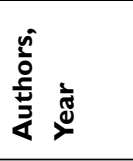 & 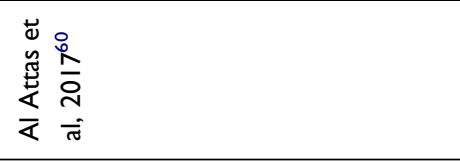 \\
\hline
\end{tabular}


molars were in patients with aggressive periodontitis and were evaluated separately. ${ }^{10,41}$ One maxillary molar in a healthy patient was treated with SRP, endodontic treatment, root resection, and periodontal regenerative surgery. ${ }^{35}$ In this case, a 6-month follow-up RVG showed decreased radiolucent areas, and a 12-month RVG follow-up demonstrated complete healing. One mandible molar did not undergo regeneration surgery and showed complete healing at 6month follow-up RVG. ${ }^{16}$ Although the small number of observed cases does not allow definitive conclusions, endoperio lesions in the mandible seem to heal faster than those in the maxilla. This preliminary assumption was confirmed by Kotze et $\mathrm{al}^{47}$ who reported a $106 \%$ higher rate of bone regeneration in the mandible compared with the maxilla. Further clinical trials are needed to prove faster regeneration rates in mandible endo-perio lesions compared with the maxilla.

Four single-rooted teeth had endo-perio lesions because of palatal radicular grooves. These cases ${ }^{42-45}$ were evaluated separately because of specific local predisposing factors. One case with PPSE in a single-rooted tooth in a diabetic patient had been previously described. ${ }^{31}$ The other seven single-rooted teeth with PPSE ${ }^{35,46}$ underwent endodontic treatment, SRP, and periodontal regenerative surgery. They showed complete healing and stability over long-term periods (1-17 years). Regenerative surgery seems to contribute to the stability of long-term outcomes for the treatment of PPSE lesions.

While there was no periodontal regenerative surgery in the present PPSE case, complete healing of the endodontic lesion and normalization of the probing depth was achieved after 6 months. This result was confirmed by Hirsch et al, ${ }^{48}$ concluding that the success rate of endoperio lesions without concomitant regenerative procedures has been reported in $27-37 \%$ of cases.

In the PPSE lesion group, four single-rooted teeth were splinted $^{35,46}$ (the diabetic patient was not counted) and two were not; ${ }^{35,42}$ over long-term periods, they had comparable results. In the present case, splinting was done before endodontic treatment, which contributed to the complete healing of the lesion in 6 months. These cases provided inconclusive information regarding the influence of splinting on tooth prognosis. This influence was also controversial in the literature. Bernal et $\mathrm{al}^{49}$ reported that splinting may not only improve the prognosis of teeth but also restore patient comfort. Graetz et al ${ }^{50}$ reported that splinting did not improve the prognosis of periodontally affected teeth. The immediate splinting of mobile single-rooted teeth with PPSE lesions seems to lead to favorable outcomes by decreasing tooth mobility and improving patient comfort.

In patients with PPSE lesions with palatal radicular grooves, ${ }^{42-45}$ the mean age was 26.5 years (minimum 19 and maximum 35). The mean age of patients with PPSE without radicular grooves was 48 years (minimum 40 and maximum 60), ${ }^{10,-16,-35,-39-46}$ considering patients with aggressive periodontitis ${ }^{10,41}$ were not counted. Thus, palatal radicular grooves are predisposing factors that provoke faster formation of endo-perio lesions and lead to endodontic therapy of intact teeth because of retrograde infections. Evaluating the probing depth in the palatal radicular groove area in each case is recommended, and, if the PD is increased, radiculoplasty might be necessary to prevent retrograde infections and endodontic treatment of intact teeth.

Two cases with palatal radicular grooves ${ }^{42,44}$ had comparable baseline parameters and underwent endodontic therapy, SRP, and periodontal regenerative surgery. One ${ }^{44}$ showed complete healing at a 6-month follow-up, and the other $^{42}$ demonstrated reduced radiolucency at a 1-year follow-up. Two cases without regenerative surgery ${ }^{43,45}$ showed reduced radiolucency at 6-month and 1-year RVG follow-ups. Such different healing periods regardless of treatment protocol may be explained by the individual periods of endodontic healing, which may take up to 4 years, ${ }^{51}$ and even later long-term healing has been reported. ${ }^{52}$ In the past, a long palatal radicular in combination with a periapical lesion often resulted in extraction of the tooth. With accurate assessment of the etiology of the defect, patient education, and a multidisciplinary approach, teeth with a palatal radicular may be retained with a stable outcome for years. ${ }^{53,54}$

Many studies indicated that combined endodontic and periodontal therapy is required for successful healing of endo-perio lesions. ${ }^{55,56}$ Schilder $^{57}$ postulated that either endodontic treatment or periodontal pathology will not produce favorable outcomes. This conclusion is controversial. While most of the analyzed cases, including the present case, received both endodontic treatment and SRP, Aksel and Serper ${ }^{16}$ reported one case of a molar with grade II mobility, grade II furcation involvement, and increased probing depth; after 1 year, complete healing was achieved after endodontic treatment with no periodontal treatment. The young age (21 years) of this systemically healthy patient likely contributed to successful healing compared with older patients with similar lesions. 
Although Lindaman ${ }^{58}$ reported that bone healing in children is faster than in adults, there are no data regarding faster healing in young adults compared with the elderly.

When endodontic procedures and non-surgical periodontal treatment fails to heal lesions, regenerative periodontal surgery or root resection may improve outcomes. ${ }^{39,59}$ This was confirmed by the case of Sharanappa et $\mathrm{al}^{37}$ in a lower molar with a $10 \mathrm{~mm}$ probing depth and grade III furcation. At a 2-month follow-up after endodontic treatment, furcation persisted, so periodontal regenerative surgery was conducted. Six months post-operatively, a $2 \mathrm{~mm}$ probing depth was achieved. Aksel and Serper ${ }^{16}$ reported a furcation lesion that persisted 3 months after endodontic treatment. The regenerative procedure was performed, and 2-year follow-up showed complete healing of the furcation.

There were three cases of endo-perio lesions in patients with aggressive periodontitis in the literature review.$10,41,60$ In all cases, the first lower molars were affected. The teeth underwent regenerative periodontal surgery and bone gain was achieved in 3, 6, 9, and 24 months. In the present case, the lower right canine was affected, which was treated using both endodontic and SRP. It healed the endodontic lesion and stabilized the marginal periodontium in 6 months. Non-surgical periodontal therapy included root scaling and planing and treatment of periodontal pockets with ozone gas. No cases in the literature review were treated using ozone therapy.

Ozone may bring a positive effect for the treatment of patients with periodontal pathology, as it was proved in recent studies that ozone antimicrobial activity and good biocompatibility with periodontal cells and gingival fibroblasts, ${ }^{61}$ ozone therapy significantly improves clinical parameters when applied in addition to periodontal therapy. ${ }^{62}$ According to Taşdemir et al, ${ }^{63}$ ozone-treated groups had higher quality-of-life due to a decrease in postoperative pain. The ozone generator which was used in the presented case is characterized by its ease of use and safety of application because of preset tissue-compatible dosages in the indication areas; it ensures a hygienic procedure during the gassing of the pockets due to its exchangeable plastic attachments. ${ }^{64}$

In patients with aggressive periodontitis, local immune mechanisms may be altered. ${ }^{65}$ Ozone therapy improves rheological properties, activates cellular metabolism, and increases intracellular ATP concentrations and expression of cytokines relevant to wound healing. ${ }^{66}$ Ramzy et al ${ }^{67}$ reported a highly significant improvement in clinical parameters and reduction of bacterial counts in quadrants treated by SRP together with ozone application compared to SRP alone in patients with aggressive periodontitis. Regarding the narrow periodontal pocket in the present case, it is important to mention that conventional cleaning of such pockets without surgical procedure are complicated and ozone gas application may have beneficial effects in such clinical condition.

Well-executed endodontic treatment is a key factor for treatment success. Poor endodontic treatment allows canal re-infection and leads to treatment failure. ${ }^{68}$ Scientists do not stop trying to improve the effectiveness of endodontic treatment using the novel techniques, such as guided endodontics, ${ }^{69}$ antimicrobial photodynamic therapy, ${ }^{70}$ and polymeric nanoparticles application for reducing dentin permeability after endodontic. ${ }^{71}$

Bacterial infections in endo-perio lesions seem to be more complicated because endodontic and periodontal pathogens may communicate through the different pathways present. ${ }^{72,73}$ According to Zehnder, ${ }^{74}$ bacteria found within root canals were present in the periodontal pocket of a tooth with endodontic problems caused by aggressive periodontal disease. In another study, Bacillus pumilus occurred in root canal systems and periodontal pockets, resulting in severe marginal periodontitis. ${ }^{75}$ Ozone is known as an agent with antibacterial effects that damage cell membranes. ${ }^{76}$ When ozone gas was combined with $2.5 \%$ sodium hypochlorite, and $2 \%$ chlorhexidine, the number of colonies of aerobic and anaerobic bacteria decreased, ${ }^{77}$ thus it is reasonable to use ozone gas in complicated cases.

Due to the variety of microbiota and increasing number of resistant strains, ${ }^{78}$ additional disinfection of root canal systems with ozone may be beneficial for patients with endo-perio lesions. From the authors clinical experience, ozone has a further positive effect that is an improvement in drying of the root canal, though further studies are necessary to prove this effect.

None of the reviewed cases utilized ozone therapy to treat endo-perio lesions. In the present case, ozone gas was added to the conventional root canal disinfection protocol for additional antibacterial effects. The efficacy of root canal disinfection was proved by the significant reduction in periapical radiolucency and beginning of bone reorganization on the lateral root surface by the complete healing at a 6-month follow-up RVG. Thus, ozone therapy may improve outcomes when treating PPSE lesions with 
narrow periodontal pockets that are difficult to access for conventional treatment without a surgical procedure.

Teeth with poor prognoses can be retained by maintaining good oral hygiene and patient compliance. ${ }^{79}$ In the present case, the patient was highly motivated to save the tooth, visited the dental hygienist regularly, and was compliant to recommended oral hygiene instructions.

Endo-perio lesions are challenging problems faced by clinicians, and, although they are relatively rare in clinical practice, they can severely compromise the tooth prognosis. They require multidisciplinary diagnosis and treatment. Regardless of the treatment protocol used, cooperation between the endodontist and periodontist is essential to treat and monitor lesion healing.

\section{Conclusion}

Using an interdisciplinary approach to treat endo-perio lesions provides favorable clinical outcomes, and proper patient oral hygiene contributes to the long-term prognosis. Additional options for root canal and periodontal pocket disinfection such as ozone gas can assist with successful outcomes of treatment endo-perio lesions with narrow periodontal pockets in patients with aggressive periodontitis and poor prognosis.

\section{Disclosure}

The authors report no conflicts of interest for this work.

\section{References}

1. Abbott PV, Salgado JC. Strategies for the endodontic management of concurrent endodontic and periodontal diseases. Aust Dent J. 2009;54: S70-85.

2. Sunitha VR, Emmandi P, Namasivayan A, Thyegarajan R, Rajaraman V. The periodontal- endodontic continuum: a review. J Conserv Dent. 2008;11(2):54-62. doi:10.4103/0972-0707.44046

3. Jivoinovici R, Suciu I, Dimitriu B, et al. Endo-periodontal lesion endodontic approach. J Med Life. 2014;7(4):542-544.

4. Rotstein I, Simon JH. Diagnosis, prognosis and decision making in treatment of combined endo-perio lesions. Periodontol. 2000;34:165203.

5. Zehnder M, Gold SI, Hasselgren G. Pathologic interaction in pulpal and periodontal tissues. J Clin Periodontol. 2002;29(8):663-671. doi:10.1034/j.1600-051X.2002.290801.x

6. Song M, Kang M, Kang DR, Jung HI, Kim E. Comparison of the effect of endodontic-periodontal combined lesion on the outcome of endodontic microsurgery with that of isolated endodontic lesion: survival analysis using propensity score analysis. Clin Oral Investig. 2018;22(4):1717-1724. doi:10.1007/s00784-017-2265-1

7. Rotstein I, Simon JH. The endo-perio lesion: a critical appraisal of the disease condition. Endod Topics. 2006;13(1):34-56. doi:10.1111/ j.1601-1546.2006.00211.x

8. Renvert S, Quirynen M. Risk indicators for peri-implantitis. A narrative review. Clin Oral Implants Res. 2015;26(Suppl 11):15-44.
9. Meric P, Buduneli N, Kanmaz B, et al. Cholinergic signalling mechanisms and early implant healing phases in healthy versus generalized aggressive periodontitis patients: a prospective, case-control study. J Clin Periodontol. 2019;46(11):1155-1163. doi:10.1111/ jcpe. 13185

10. Kavarthapu A, Malaiappan S. Management of periodontic-endodontic lesion in aggressive periodontitis-9 months follow-up: report of a case. Indian J Dent Res. 2019;30:149-153.

11. Pjetursson BE, Thoma D, Jung R, Zwahlen M, Zembic A. A systematic review of the survival and complication rates of implantsupported fixed dental prostheses (FDPs) after a mean observation period of at least 5 years. Clin Oral Implants Res. 2012;23(Suppl 6):22-38. doi:10.1111/j.1600-0501.2012.02546.x

12. Herrera D, Retamal-Valdes B, Alonso B, Feres M. Acute periodontal lesions (periodontal abscesses and necrotizing periodontal diseases) and endo-periodontal lesions. J Periodontol. 2018;89(1):S85-S102. doi:10.1002/jper.16-0642

13. Armitage GC. Development of a classification system for periodontal diseases and conditions. Ann Periodontol. 1999;4(1):1-6. doi:10.1902/annals.1999.4.1.1

14. Hany MAA. Different perspectives in understanding he pulp and periodontal intercommunications with a new proposed classification for endo-perio lesions. ENDO (Lond Engl). 2012;6(2):87-104.

15. Al-Fouzan KS. A new classification of endodontic periodontal lesions. Int J Dent. 2014;2014:5. doi:10.1155/2014/919173

16. Aksel H, Serper A. Serper A case series associated with different kinds of endo-perio lesions. J Clin Exp Dent. 2014;6(1):e91-e95. doi: $10.4317 /$ jced.51219

17. Greene JG, Vermillion JR. The simplified oral hygiene index. J Am Dent Assoc. 1964;68(1):7-13. doi:10.14219/jada.archive.1964.0034

18. ISO 3950. Dentistry-Designation System for Teeth and Areas of the Oral Cavity. Switzerland: ISO; 2009.

19. British Society of Periodontology. Basic periodontal examination (BPE); 2016. Available from: http://www.bsperio.org.uk/publica tions/downloads/39_150345_bpe-2016-po-v5-final.pdf.

20. ICD -10 version; 2019. Available from: https://icd.who.int/ browse10/2019/en\#/K00-K14. Accessed September 26, 2020.

21. Byakova SF, Novozhilova NE, Makeeva IM, Grachev VI, Kasatkina IV. The accuracy of CBCT for the detection and diagnosis of vertical root fractures in vivo. Int Endod J. 2019;52(9):1255-1263. doi:10.1111/iej.13114

22. Bender IB, Seltzer S. The effect of periodontal disease on the pulp. Oral Surg Oral Med Oral Pathol Oral Radiol Endod. 1972;33 (3):458-474. doi:10.1016/0030-4220(72)90476-8

23. Alquthami H, Almalik AM, Alzahrani FF, Badawi L. Successful management of teeth with different types of endodontic-periodontal lesions. Case Rep Dent. 2018;7084245. doi:10.1155/2018/7084245

24. Bhide A, Shah PS, Acharya G. A simplified guide to randomized controlled trials. Acta Obstet Gynecol Scand. 2018;97(4):380-387. doi:10.1111/aogs.13309

25. Singh P. Endo-perio dilemma: a brief review. Dent Res J (Isfahan). 2011;8(1):39-47.

26. Oh SL, Fouad AF, Park SH. Treatment strategy for guided tissue regeneration in combined endodontic-periodontal lesions: case report and review. $J$ Endod. 2009;35(10):1331-1336. doi:10.1016/j. joen.2009.06.004

27. Gupta S, Tewari S, Tewari S, Mittal S. Effect of time lapse between endodontic and periodontal therapies on the healing of concurrent endodontic-periodontal lesions without communication: a prospective randomized clinical trial. J Endod. 2015;41(6):785-790. doi:10.1016/ j.joen.2015.02.015

28. Schidt JC, Walter C, Amato M, Weiger R. Treatment of periodontalendodontic lesions-a systematic review. J Clin Periodontol. 2014;41 (8):779-790. doi:10.1111/jcpe. 12265

29. Abbott PJ. Endodontic management of combined endodontic-periodontal lesions. NZ Soc Periodontol. 1998;(83):15-28. 
30. Goyal L, Gupta N, Gupta ND. Autologous platelet-rich derivatives along with alloplastic bone substitute in the management of complex perio-endo cases. J Indian Soc Periodontol. 2020;24(2):182-185. doi:10.4103/jisp.jisp_81_19

31. Dhoum S, Laslami K, Rouggani F, El Ouazzani A, Jabri M. Endoperio lesion and uncontrolled diabetes. Case Rep Dent. 2018;2018:7478236. doi:10.1155/2018/7478236

32. Gomes MS, Blattner TC, Sant'Ana Filho M, et al. Can apical periodontitis modify systemic levels of inflammatory markers? A systematic review and meta-analysis. J. Endod. 2013;39(10):1205-1217. doi:10.1016/j.joen.2013.06.014

33. Ishihara K, Ando T, Kosugi M, et al. Relationship between the onset of pustulosis palmaris et plantaris, periodontitis and bacterial heat shock proteins. Oral Microbiol Immunol. 2000;15(4):232-237. doi:10.1034/j.1399-302x.2000.150404.x

34. de Pablo P, Chapple IL, Buckley CD, Dietrich T. Periodontitis in systemic rheumatic diseases. Nat Rev Rheumatol. 2009;5(4):218224. doi:10.1038/nrrheum.2009.28

35. Pico-Blanco A, Casteli-Baz P, Caneiro-Queija L, Liňares-Gonzá A, Martin-Lacharro P, Blanco-Carrión J. Saving single-rooted teeth with combined endodontic-periodontal lesions. $J$ Endod. 2016;42 (12):1859-1864. doi:10.1016/j.joen.2016.08.015

36. Jivoinovici R, Suciu I, Gheorghiu I, Suciu I. Clinical radiological aspects of primary endodontic lesions with secondary periodontal involvement. $J$ Med Life. 2017;10(1):70-75.

37. Kambale S, Aspalli N, Munavalli A, Ajgaonkar N, Babannavar R. A sequential approach in treatment of endo-perio lesion. $J$ Clin Diagn Res. 2014;8(8):ZD22-ZD24. doi:10.7860/JCDR/2014/9927.4692

38. Goyal L. Clinical effectiveness of combining platelet rich fibrin with alloplastic bone substitute for the management of combined endodontic periodontal lesion. Restor Dent Endod. 2014;39(1):51-55. doi:10.5395/rde.2014.39.1.51

39. Varughese V, Mahendra J, Thomas AR, Ambalavanan N. Resection and regeneration - a novel approach in treating a perio-endo lesion. $J$ Clin Diagn Res. 2015;9(3):ZD08-10. doi:10.7860/JCDR/2015/11096.5643

40. Makino-Oi A, Ishii Y, Makino K, et al. Treatment of severe chronic periodontitis with surgical and prosthetic intervention: a 9-year follow-up case report. Bull Tokyo Dent Coll. 2017;58(4):237-246. doi:10.2209/tdcpublication.2016-0043

41. Fahmy MD, Luepke PG, Ibrahim MS, Guentsch A. Treatment of a periodontic-endodontic lesion in a patient with aggressive periodontitis. Case Rep Dent. 2016;2016:7080781. doi:10.1155/2016/7080781

42. Nadig PP, Agrawal IS, Agrawal VS, Srinivasan SC. Palato-radicular groove: a rare entity in maxillary central incisor leading to endo-perio lesion. J Clin Diagn Res. 2016;10(8):ZJ14-ZJ15.

43. Gandhi A, Kathuria A, Gandhi T. Endodontic-periodontal management of two rooted maxillary lateral incisor associated with complex radicular lingual groove by using spiral computed tomography as a diagnostic aid: a case report. Int Endod J. 2011;44(6):574-582. doi:10.1111/j.1365-2591.2011.01868.x.

44. Praveena Devi B, Das A, Kundu R, Bagchi S, Pal AK. The silent killer caught white handed: an interdisciplinary approach to manage a rare case of PGG associated perio-endo lesion. Int J Appl Dent Sci. 2019;5(1):01-04.

45. Naik M, de Ataide Ide N, Fernandes M, Lambor R. Treatment of combined endodontic: periodontic lesion by sealing of palato-radicular groove using biodentine. J Conserv Dent. 2014;17(6):594-597. doi:10.4103/0972-0707.144613

46. Kumar KA, Chakravarthy M, Selvarajan S, Ramakrishnan T, Ari G. Use of an amniotic membrane as a novel barrier in a tooth with a questionable prognosis. J Indian Soc Periodontol. 2017;21(3):237240. doi:10.4103/jisp.jisp_67_16

47. Kotze MJ, Bütow K, Olorunju SA, et al. A comparison of mandibular and maxillary alveolar osteogenesis over six weeks: a radiological examination. Head Face Med. 2014;10(1):50. doi:10.1186/1746$160 \mathrm{X}-10-50$
48. Hirsch JM, Ahlström U, Henrikson PA, Heyden G, Peterson LE. Periapical surgery. Int $J$ Oral Surg. 1979;8(3):173-185. doi:10.1016/S0300-9785(79)80016-2

49. Bernal G, Carvajal JC, Muňoz-Viveros CA. A review of the clinical management of mobile teeth. J Contemp Dent Pract. 2002;3(4):1022. doi: $10.5005 /$ jcdp-3-4-10

50. Graetz C, Ostermann F, Woeste S, Sälzer S, Dörfer CE, Schwendicke F. Long-term survival and maintenance efforts of splinted teeth in periodontitis patients. $J$ Dent. 2019;80:49-54. doi:10.1016/j. jdent.2018.10.009

51. Setzer FC, Kim S. Comparison of long-term survival of implants and endodontically treated teeth. $J$ Dent Res. 2014;93(1):19-26. doi:10.1177/0022034513504782

52. Molven O, Halse A, Fristad I, MacDonald-Jankowski D. Periapical changes following root-canal treatment observed 20-27 years postoperatively. Int Endod. 2002;J35(9):784. doi:10.1046/j.13652591.2002.00568.x

53. Katwal D, Fiorica JK, Bleuel J, Clark SJ. Successful multidisciplinary management of an endodontic-periodontal lesion associated with a palato-radicular groove: a case report. Clin Adv Periodontics. 2020;10(2):88-93. doi:10.1002/cap.10079

54. Ustaoğlu G, Uğur Aydin Z, Özelçi F. Comparison of GTR, T-PRF and open-flap debridement in the treatment of intrabony defects with endo-perio lesions: a randomized controlled trial. Med Oral Patol Oral Cir Bucal. 2020;25(1):e117-e123. doi:10.4317/ medoral.23231

55. Hiatt W, Amen C. Periodontal pocket elimination by combined therapy. Dent Clin North Am. 1964;133:44.

56. Storrer CM, Bordin GM, Pereira TT. How to diagnose and treat periodontal endodontic lesions? RSBO. 2012;9:427-433.

57. Schilder H. Endodontic-periodontal therapy. In: Grossman L, editor. Endodontic Practice. 6th ed. Philadelphia: Lea and Febiger; 1965.

58. Lindaman LM. Bone healing in children. Clin Podiatr Med Surg. 2001;18(1):97-108.

59. Torabinejad M, Trope M. Principles and practice of endodontics. In: Walton RE, Torabinejad M, editors. Principles and Practice of Endodontics. 2 ed. Philodelphia: W.B. Saunders company; 1996:452-455.

60. Al Attas MA, Edrees HY, Aya MN, Sammani AA. Madarati multidisciplinary management of concomitant pulpal and periodontal lesion: a case report. J Taibah Univ Med Sci. 2017;12(5):455-460.

61. Moraschini V, Kischinhevsky ICC, Calasans-Maia MD, et al. Ineffectiveness of ozone therapy in nonsurgical periodontal treatment: a systematic review and metaanalysis of randomized clinical trials. Clin Oral Investig. 2020;24(6):1877-1888. doi:10.1007/ s00784-020-03289-2

62. Talmaç AC, Çalişir M. Efficacy of gaseous ozone in smoking and non-smoking gingivitis patients. Ir J Med Sci. 2020. doi:10.1007/ s11845-020-02271-x

63. Taşdemir Z, Alkan BA, Albayrak H. Effects of ozone therapy on the early healing period of deepithelialized gingival grafts: a randomized placebo-controlled clinical trial. J Periodontol. 2016;87(6):663-671. doi:10.1902/jop.2016.150217

64. Gupta G, Mansi B. Ozone therapy in periodontics. J Med Life. 2012;5 (1):59-67.

65. Emingil G, Atilla G, Hüseyinov A. Gingival crevicular fluid monocyte chemoattractant protein-1 and RANTES levels in patients with generalized aggressive periodontitis. J Clin Periodontol. 2004;31 (10):829-834. doi:10.1111/j.1600-051X.2004.00584.x

66. Patel PV, Kumar V, Kumar S, Vidya GD, Patel A. Therapeutic effect of topical ozonated oil on the epithelial healing of palatal wound sites: a planimetrical and cytological study. J Investig Clin Dent. 2011;2(4):248-258. doi:10.1111/j.2041-1626.2011.00072.x

67. Ramzy MI, Gomaa HE, Mostafa MI. Management of aggressive periodontitis using ozonized water. Egypt Med J NRC. 2005;6:229245 . 
68. Peters LB, Wesselink PR, Moorer WR. The fate and the role of bacteria left in root dentinal tubules. Int Endod J. 1995;28(2):9599. doi:10.1111/j.1365-2591.1995.tb00166.x

69. Moreno-Rabié C, Torres A, Lambrechts P, Jacobs R. Clinical applications, accuracy and limitations of guided endodontics: a systematic review. Int Endod J. 2020;53(2):214-231. doi:10.1111/iej.13216

70. Bordea IR, Hanna R, Chiniforush N, et al. Evaluation of the outcome of various laser therapy applications in root canal disinfection: a systematic review. PhotodiagnosisPhotodynTher. 2020;29:101611. doi:10.1016/j.pdpdt.2019.101611

71. Toledano M, Osorio E, Aguilera FS, et al. Polymeric nanoparticles for endodontic therapy. $J$ Mech Behav Biomed Mater. 2020;103:103606. doi:10.1016/j.jmbbm.2019.103606

72. Kerekes K, Olsen I. Similarities in the microfloras of root canals and deep periodontal pockets. Endod Dent Traumatol. 1990;6(1):1-5. doi:10.1111/j.1600-9657.1990.tb00378.x

73. Das AC, Sahoo SK, Parihar AS, Bhardwaj SS, Babaji P, Varghese JG. Evaluation of role of periodontal pathogens in endodontic periodontal diseases. J Family Med Prim Care. 2020;9(1):239-242. doi:10.4103/ jfmpc.jfmpc_725_19
74. Zehnder M. Endodontic infection caused by localized aggressive periodontitis: a case report and bacteriologic evaluation. Oral Surg Oral Med Oral Pathol Oral Radiol Endod. 2001;92(4):440-445. doi:10.1067/moe.2001.117270

75. Johnson BT, Shaw LN, Nelson DC, Ja M. Extracellular proteolytic activities expressed by Bacillus pumilus isolated from endodontic and periodontal lesions. J Med Microbiol. 2008;57(5):643-651. doi:10.1099/ jmm.0.47754-0

76. German IJS, Rodrigues ADC, Andreo JC. Ozone therapy in dentistry. A systematic review. Int $J$ Odontostomat. 2013;7(2):267-278. doi:10.4067/S0718-381X2013000200017

77. Ajeti NN, Pustina-Krasniqi T, Apostolska S. The effect of gaseous ozone in infected root canal open access maced. J Med Sci. 2018;6(2):389-396.

78. Nair PN. Pathogenesis of Apical periodontitis and the causes of endodontic failures. Crit Rev Oral Biol Med. 2004;15(6):348-381. doi: $10.1177 / 154411130401500604$

79. Axelsson P, Nyström B, Lindhe J. The long-term effect of a plaque control program on tooth mortality, caries and periodontal disease in adults. Results after 30 years of maintenance. J Clin Periodontol. 2004;31(9):749-757. doi:10.1111/j.1600-051X.2004.00563.x

\section{Publish your work in this journal}

Clinical, Cosmetic and Investigational Dentistry is an international, peer-reviewed, open access, online journal focusing on the latest clinical and experimental research in dentistry with specific emphasis on cosmetic interventions. Innovative developments in dental materials, techniques and devices that improve outcomes and patient satisfaction and preference will be highlighted. The manuscript management system is completely online and includes a very quick and fair peer-review system, which is all easy to use. Visit http://www.dovepress.com/testimonials.php to read real quotes from published authors. 